\title{
Electron Spin Relaxation and Biochemical Characterization of the Hydrogenase Maturase HydF: Insights into [2Fe-2S] and [4Fe-4S] Cluster Communication and Hydrogenase Activation
}

Eric M. Shepard, ${ }^{\dagger}$ Amanda S. Byer, ${ }^{\dagger}$ Priyanka Aggarwal, ${ }^{\dagger}$ Jeremiah N. Betz, ${ }^{\dagger}$ Anna G. Scott, ${ }^{\dagger}$ Krista A. Shisler, ${ }^{\dagger}$ Robert J. Usselman, ${ }^{\dagger}$ Gareth R. Eaton, ${ }^{+}$Sandra S. Eaton, ${ }^{\ddagger}$ and Joan B. Broderick ${ }^{*}{ }^{\dagger}$

${ }^{\dagger}$ Department of Chemistry and Biochemistry, Montana State University, Bozeman, Montana 59717, United States

${ }^{\ddagger}$ Department of Chemistry and Biochemistry, University of Denver, Denver, Colorado 80208, United States

\section{Supporting Information}

\begin{abstract}
Nature utilizes [FeFe]-hydrogenase enzymes to catalyze the interconversion between $\mathrm{H}_{2}$ and protons and electrons. Catalysis occurs at the H-cluster, a carbon monoxide-, cyanide-, and dithiomethylamine-coordinated $2 \mathrm{Fe}$ subcluster bridged via a cysteine to a $[4 \mathrm{Fe}-4 \mathrm{~S}]$ cluster. Biosynthesis of this unique metallocofactor is accomplished by three maturase enzymes denoted HydE, HydF, and HydG. HydE and HydG belong to the radical $S$-adenosylmethionine superfamily of enzymes and synthesize the nonprotein ligands of the H-cluster. These enzymes interact with HydF, a GTPase that acts as a scaffold or carrier protein during $2 \mathrm{Fe}$ subcluster assembly. Prior characterization of HydF demonstrated the protein exists in both dimeric and tetrameric states and coordinates both $[4 \mathrm{Fe}-4 \mathrm{~S}]^{2+/+}$ and $[2 \mathrm{Fe}-2 \mathrm{~S}]^{2+/+}$ clusters [Shepard, E. M., Byer, A. S., Betz, J. N., Peters, J. W., and Broderick, J. B. (2016) Biochemistry 55, 3514-3527]. Herein, electron paramagnetic resonance $(\mathrm{EPR})$ is utilized to characterize the $[2 \mathrm{Fe}-2 \mathrm{~S}]^{+}$and $[4 \mathrm{Fe}-4 \mathrm{~S}]^{+}$clusters bound to HydF. Examination of spin relaxation times using pulsed EPR in HydF samples exhibiting both $[4 \mathrm{Fe}-4 \mathrm{~S}]^{+}$and $[2 \mathrm{Fe}-2 \mathrm{~S}]^{+}$cluster EPR signals supports a model in which the two cluster types either are bound to widely separated sites on HydF or are not simultaneously bound to a single HydF species. Gel filtration chromatographic analyses of $\mathrm{HydF}$ spectroscopic samples strongly suggest the $[2 \mathrm{Fe}-2 \mathrm{~S}]^{+}$and $[4 \mathrm{Fe}-4 \mathrm{~S}]^{+}$clusters are coordinated to the dimeric form of the protein. Lastly, we examined the $2 \mathrm{Fe}$ subcluster-loaded form of HydF and showed the dimeric state is responsible for [FeFe]-hydrogenase activation. Together, the results indicate a specific role for the HydF dimer in the H-cluster biosynthesis pathway.
\end{abstract}

$[\mathrm{FeFe}]$-hydrogenase and $[\mathrm{NiFe}]$-hydrogenase are two evolutionarily unrelated enzyme families responsible for catalyzing the majority of $\mathrm{H}_{2}$ metabolism in biology. These two enzyme families share similar active site morphology as evidenced by the presence of iron-bound carbon monoxide (CO) and cyanide $\left(\mathrm{CN}^{-}\right)$ligands. ${ }^{1}[\mathrm{FeFe}]$ - and $[\mathrm{NiFe}]$-hydrogenases exhibit different $\mathrm{O}_{2}$ tolerances, and it is the $[\mathrm{FeFe}]$-hydrogenase class that is predominantly responsible for $\mathrm{H}_{2}(\mathrm{~g})$ evolution with turnover rates of $10^{4}$ events per second. ${ }^{2-7}$ In $[\mathrm{FeFe}$ hydrogenase (HydA) enzymes, the active site metallocofactor is a $6 \mathrm{Fe}$ entity, known as the $\mathrm{H}$-cluster, that involves a standard [4Fe-4S] cubane linked through a cysteine thiolate to a diiron subcluster; ligands to the two Fe ions of the subcluster include three $\mathrm{CO}$ molecules, two $\mathrm{CN}^{-}$ions, and a bridging dithiomethylamine (DTMA) (Figure 1$).^{8-11}$

In conjunction with the ISC (iron-sulfur cluster assembly) machinery of the cellular environment, biology utilizes three proteins denoted HydE, HydF, and HydG to accomplish the biosynthesis of the $\mathrm{H}$-cluster. The heterologous expression of active HydA in Escherichia coli is accomplished by co-expression of the $[\mathrm{FeFe}]$-hydrogenase with $\mathrm{HydE}, \mathrm{HydF}$, and HydG. ${ }^{12-14}$ The classification of $\mathrm{HydE}$ and $\mathrm{HydG}$ as radical $S$ - adenosylmethionine (SAM) enzyme superfamily members establishes a parallel between $\mathrm{H}$-cluster synthesis and nitrogenase complex metallocofactor biosynthesis, while also further distinguishing it from [NiFe]-hydrogenase active site biosynthesis. ${ }^{1,12,15-18}$ Radical SAM enzymes utilize the unique Fe site of a site-differentiated, redox-active [4Fe-4S] cluster to anchor SAM through its carboxylate and amine groups; this interaction leads to inner sphere electron transfer from the $[4 \mathrm{Fe}-4 \mathrm{~S}]$ cluster into SAM's sulfonium group, ultimately generating a $5^{\prime}$ deoxyadenosyl radical $\left(5^{\prime}\right.$-dAdo $\left.{ }^{\circ}\right)$ species that performs a spectacular array of substrate-derived $\mathrm{H}$ atom abstraction events. $^{19}$

While several studies of $[\mathrm{FeFe}]$-hydrogenase maturation suggest the absolute requirement for $\mathrm{HydE}$ in the maturation process, ${ }^{12,13}$ this enzyme's exact role in H-cluster biosynthesis has remained elusive. We demonstrated that the HydEcatalyzed cleavage of SAM is stimulated by select thiolcontaining compounds, which also exhibit significant effects on

Received: February 23, 2017

Revised: May 18, 2017

Published: May 19, 2017 

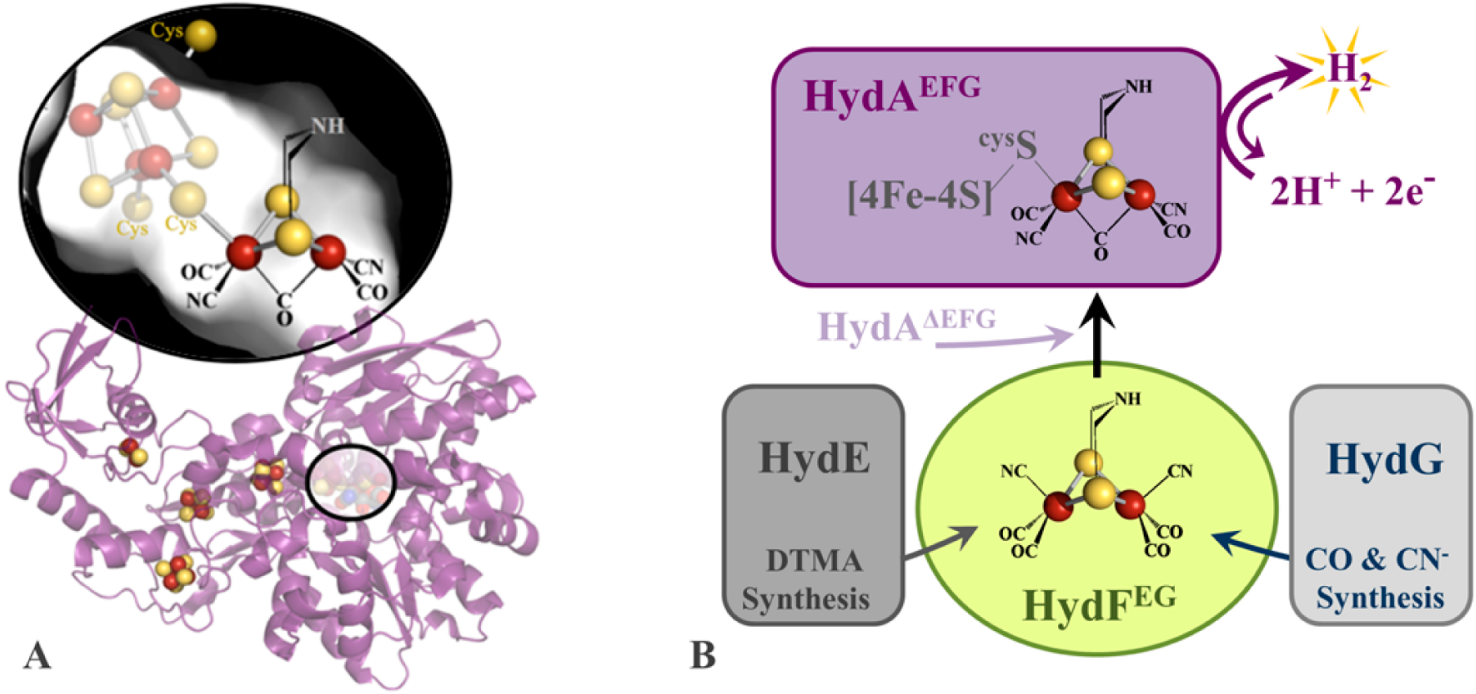

Figure 1. [FeFe]-Hydrogenase and H-cluster maturation. (A) [FeFe]-Hydrogenase from Clostridium pasteurianum I (CpI) (PDB entry 3C8Y). The oval highlights the catalytic $\mathrm{H}$-cluster. Below the oval, a cartoon of the crystal structure is colored purple with the accessory FeS clusters and the $\mathrm{H}$ cluster all shown as spheres. (B) Hypothetical maturation scheme for the biosynthesis of the $2 \mathrm{Fe}$ subcluster $\mathrm{H}$-cluster precursor on HydF (see the text for additional details). The color scheme for the FeS clusters depicted in this figure is as follows: iron, rust; sulfur, yellow.

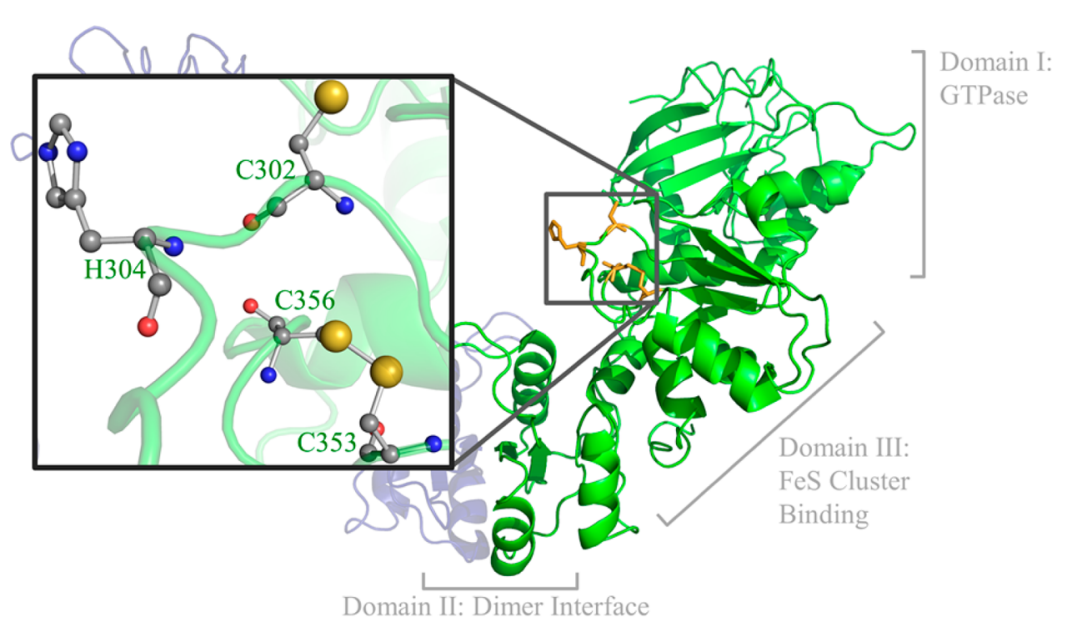

Figure 2. HydF structure. The crystal structure of the HydF dimer (PDB entry 3QQ5) is depicted with one subunit colored transparent dark blue and the other bright green. Domains I-III are approximately defined and labeled in gray. The conserved residues that are expected to bind the FeS cluster(s) are depicted as sticks and colored gold in the green subunit. The close-up highlights the proximity of the conserved histidine and cysteine residues that are shown as sticks and spheres. Residues that are involved in binding and hydrolyzing GTP are located in domain I. The color scheme for this figure is as follows: carbon, gray; nitrogen, blue; oxygen, red; sulfur, yellow.

the amount of deuterium atom incorporated into 5'deoxyadenosine from $\mathrm{D}_{2} \mathrm{O} .{ }^{20}$ Drawing from in vitro synthesis, we hypothesized that HydE-based $\mathrm{C} \alpha-\mathrm{C} \beta$ bond cleavage of two thiol-containing species could generate two thioformaldehyde molecules that can condense with ammonia to produce the bridging DTMA ligand (Figure 1). ${ }^{20,21}$ Recent X-ray crystallographic studies made the unexpected discovery that the $5^{\prime}$-dAdo ${ }^{*}$ radical could attack $(2 R, 4 R)$-2-methyl-1,3-thiazolidine-2,4-dicarboxylic acid to form a new 5 -C-sulfur bond in the $S$-adenosylcysteine product. ${ }^{22}$ Despite the ability of HydE to react directly with the sulfur atom in 1,3-thiazoldine compounds, these molecules do not appear to be this enzyme's physiological substrate because of their inability to enhance activation of $[\mathrm{FeFe}]$-hydrogenase. ${ }^{22}$

While HydE's natural substrate and chemical reaction remain unclear, substantial insight into HydG's role in H-cluster maturation has been provided by several laboratories. HydG contains two distinct FeS cluster motifs located at opposite ends of the TIM barrel structure. ${ }^{23,24}$ At the N-terminal radical SAM [4Fe-4S] cluster, substrate tyrosine is activated by reversible $\mathrm{H}$ atom abstraction and decomposed into $p$-cresol and dehydroglycine (DHG); ${ }^{25-28}$ the DHG fragment is the source of the diatomic species $\mathrm{CN}^{-}$and $\mathrm{CO}$ (Figure 1)..$^{26,28-31}$ Recent spectroscopic and structural work has shown that the accessory cluster site in HydG is occupied by a $[4 \mathrm{Fe}-4 \mathrm{~S}]\left[\left(\kappa^{3}-\right.\right.$ Cys)Fe] moiety that is proposed to be the site of $\mathrm{Fe}(\mathrm{CO})_{2}(\mathrm{CN}) \mathrm{Cys}$ synthon formation, although the precise mechanistic details of DHG breakdown at this site require further clarification. ${ }^{24,28,32-36}$

HydF contains Walker A P-loop and Walker $\mathrm{B} \mathrm{Mg}^{2+}$ binding motifs common to small Ras GTPase enzymes ${ }^{37}$ and additionally includes a C-terminal $\mathrm{CXHX}_{46-53} \mathrm{CXXC}$ motif responsible for coordination of an FeS cluster; these protein motifs are absolutely necessary for achieving active HydA. ${ }^{13}$ 
The apo crystal structure of Thermotoga neopolitana (T.n.) HydF has been determined to $2.99 \AA$ resolution. ${ }^{38}$ The HydF monomer is comprised of three domains. Domain I contains the residues responsible for coordinating and hydrolyzing GTP. Domain II is the dimerization domain and is responsible for stabilizing the dimeric state via a $1800 \AA$ buried surface and a four-stranded parallel $\beta$-sheet bridging the two monomers. Domain III contains the $\mathrm{CXHX}_{46-53} \mathrm{CXXC}$ motif, which is located approximately $35 \AA$ from the GTP binding site (Figure 2).

HydF's GTPase activity has been demonstrated in both Thermotoga maritima (T.m.) and Clostridium acetobutylicum (C.a.) proteins, and preliminary evidence suggests that GTP binding acts as a molecular switch in gating the protein-protein interactions between $\mathrm{HydF}$ and $\mathrm{HydE} / \mathrm{HydG}$ during the maturation process. ${ }^{31,39-42}$ Insight into the role HydF plays during H-cluster assembly was provided by biochemical characterization of the heterologously expressed and purified C.a. HydF protein $\left(\mathrm{HydF}^{\Delta \mathrm{EG}}\right)$ relative to the purified C.a. HydF protein that was co-expressed in a genetic background with $\mathrm{HydE}$ and $\mathrm{HydG}\left(\mathrm{HydF}^{\mathrm{EG}}\right) ;{ }^{40,43}$ importantly, $\mathrm{HydF}^{\mathrm{EG}}$ exhibits the ability to activate purified HydA when the latter is expressed in the absence of HydE, HydF, and HydG $\left(\mathrm{HydA}^{\Delta \mathrm{EFG}}\right){ }^{43,44}$ Moreover, FTIR analysis of purified $\mathrm{HydF}^{\mathrm{EG}}$ shows $\mathrm{Fe}-\mathrm{CO}$ and $\mathrm{Fe}-\mathrm{CN}^{-}$species, ${ }^{40}$ a result further substantiated by similar spectroscopic studies using homologously overexpressed C.a. $\mathrm{HydF}^{\mathrm{EG}} .{ }^{45}$ On the other hand, $\mathrm{HydF}^{\mathrm{EGG}}$ contains neither $\mathrm{Fe}-\mathrm{CO}$ nor $\mathrm{Fe}-\mathrm{CN}^{-}$spectroscopic features, nor can it activate $\mathrm{HydA}^{\Delta \mathrm{EFG}}{ }^{40}$ Together, these results support a model in which HydE and HydG interact with HydF to assemble the $2 \mathrm{Fe}$ subcluster of the H-cluster on HydF (Figure 1B). Additional experimental support for a scaffold/ carrier hypothesis has been provided by studies that show $\mathrm{HydA}^{\triangle \mathrm{EFG}}$ can be activated by HydF that has been loaded with synthetic $2 \mathrm{Fe}$ subcluster analogues. ${ }^{11,46}$ Further experimentation is needed to clarify HydF's precise role, including whether $\mathrm{HydF}$ provides the iron or sulfur components of the $2 \mathrm{Fe}$ subcluster or whether all components are delivered by the actions of HydE and HydG.

Our recent spectroscopic characterization of HydF using ultraviolet-visible (UV-vis), circular dichroism (CD), and EPR techniques demonstrated this protein coordinates redoxactive $[2 \mathrm{Fe}-2 \mathrm{~S}]$ and $[4 \mathrm{Fe}-4 \mathrm{~S}]$ clusters. ${ }^{47}$ The biosynthetic role of the HydF-coordinated [2Fe-2S] cluster is unresolved, but it has been suggested that it is a possible delivery site for the small molecule ligand products synthesized by $\mathrm{HydE}$ and $\mathrm{HydG}$ during $2 \mathrm{Fe}$ subcluster assembly. ${ }^{31,40,47}$ Herein, we examine the $[2 \mathrm{Fe}-2 \mathrm{~S}]^{+}$cluster-coordinated state of $\mathrm{HydF}$ and examine whether the $[2 \mathrm{Fe}-2 \mathrm{~S}]$ cluster is bridged to the $[4 \mathrm{Fe}-4 \mathrm{~S}]$ cluster using pulsed EPR and relaxation enhancement experiments. Gel filtration chromatography is used to provide a picture of the quaternary structural state of the HydF protein in the EPR samples. These approaches allow us to draw conclusions regarding whether the different quaternary forms of HydF coordinate distinct iron-sulfur clusters. Moreover, we demonstrate that $\mathrm{HydA}^{\triangle \mathrm{EFG}}$ activation is accomplished by the dimeric state of $\mathrm{HydF}^{\mathrm{EG}}$. Together, these results not only provide a more thorough picture of the iron-sulfur cluster states associated with $\mathrm{HydF}$ prior to interaction with $\mathrm{HydE}$ and HydG but also help to define the pathway via which loaded $\mathrm{HydF}^{\mathrm{EG}}$ is produced.

\section{EXPERIMENTAL PROCEDURES}

Expression and Purification of HydF. $\mathrm{HydF}^{\Delta \mathrm{EG}}$ and $\mathrm{HydF}^{\mathrm{EG}}$ proteins were overexpressed, grown, and purified with very minor alterations to our previously described protocols. $^{43,47}$ The genes for C.a. hydE, hydF (containing an $\mathrm{N}$ terminal six-histidine tag), and hydG were cloned into pETDuet, pRSFDuet, and pCDFDuet vectors, respectively. For expression of the $\mathrm{HydF}^{\Delta \mathrm{EG}}$ protein, only the hydF construct was transformed into E. coli BL21(DE3) (Stratagene) cells. For expression of the $\mathrm{HydF}^{\mathrm{EG}}$ protein, all three of the constructs mentioned above were transformed into E. coli BL21(DE3)RIL, BL21(DE3)pLysS, or BL21(DE3) (Stratagene) cells, wherein all proteins are overexpressed in similar amounts.

The transformed constructs were used to streak fresh LBagar antibiotic plates; single colonies from these plates were chosen for pilot scale overnight culture growth. The following morning the pilot cultures were used to inoculate $9 \mathrm{~L}$ of medium that contained $10 \mathrm{~g} / \mathrm{L}$ tryptone, $5 \mathrm{~g} / \mathrm{L}$ yeast extract, 5 $\mathrm{g} / \mathrm{L} \mathrm{KCl}, 5 \mathrm{~g} / \mathrm{L}$ glucose, and $50 \mathrm{mM}$ potassium phosphate buffer $(\mathrm{pH} 7.5)$, supplemented with the appropriate antibiotics. All cell cultures were grown at $37{ }^{\circ} \mathrm{C}$ with $230 \mathrm{rpm}$ shaking, induced with $1 \mathrm{mM}$ isopropyl $\beta$-D-1-thiogalactopyranoside, and supplemented with $0.16 \mathrm{mM}$ ferrous ammonium sulfate at $\mathrm{OD}_{600}$ values of $0.45-0.55$. Cultures were allowed to continue to grow at $37{ }^{\circ} \mathrm{C}$ and $230 \mathrm{rpm}$ for $2.5 \mathrm{~h}$, at which time they were cooled to room temperature prior to addition of a final aliquot of ferrous ammonium sulfate $(0.16 \mathrm{mM})$. Cultures were then sparged with $\mathrm{N}_{2}(\mathrm{~g})$ for $\sim 15 \mathrm{~h}$ at $4{ }^{\circ} \mathrm{C}$; cells were harvested by centrifugation, and pellets were flash-frozen in liquid $\mathrm{N}_{2}$ and stored at $-80{ }^{\circ} \mathrm{C}$ until they were used further.

All cell lysis and purification techniques were performed in a Coy (Grass Lake, MI) anaerobic chamber maintained with an approximately $96 \% \mathrm{~N}_{2}(\mathrm{~g}), 4 \% \mathrm{H}_{2}(\mathrm{~g})$ atmosphere that was housed inside a $4{ }^{\circ} \mathrm{C}$ walk-in refrigerator. Frozen cell pellets were resuspended in a lysis buffer containing $50 \mathrm{mM}$ HEPES ( $\mathrm{pH} 7.4), 0.3 \mathrm{M} \mathrm{KCl}, 5 \%$ glycerol, and $10 \mathrm{mM}$ imidazole (buffer A). The lysis buffer was supplemented with $10 \mathrm{mM}$ $\mathrm{MgCl}_{2}, 1 \mathrm{mM}$ PMSF, $0.5 \%$ Triton X-100, $0.07 \mathrm{mg}$ of DNase and RNase per gram of cells, and $\sim 0.4 \mathrm{mg}$ of lysozyme per gram of cells; the mixture was stirred for $10 \mathrm{~min}$ and then subjected to sonication ( $5 \mathrm{~min}$ total pulse time at $60 \%$ amplitude) using a model FB505 sonic dismembrator (500 W, Fisher Scientific). The lysate was centrifuged at $18000 \mathrm{rpm}$ for $30 \mathrm{~min}$, and the supernatant was loaded onto a $5 \mathrm{~mL}$ HisTrap $\mathrm{Ni}^{2+}$-affinity column (GE Healthcare) using an $\ddot{\mathrm{KKTA}}$ Basic 100 FPLC instrument (GE Healthcare). All subsequent wash and elution steps [using a $50 \mathrm{mM}$ HEPES ( $\mathrm{pH} 7.4), 0.3 \mathrm{M} \mathrm{KCl}$, $5 \%$ glycerol, $500 \mathrm{mM}$ imidazole buffer] were performed as previously described, to isolate and purify only the His-tagged HydF protein. ${ }^{47}$ "Freshly purified" HydF denotes protein eluting at $50 \%$ buffer B with a buffer consisting of $50 \mathrm{mM}$ HEPES ( $\mathrm{pH} 7.4$ ), $0.3 \mathrm{M} \mathrm{KCl}, 5 \%$ glycerol, and $255 \mathrm{mM}$ imidazole. "As-isolated" HydF denotes protein that was either dialyzed or buffer exchanged into a $50 \mathrm{mM}$ HEPES ( $\mathrm{pH} 7.4$ ), $0.3 \mathrm{M} \mathrm{KCl}, 5 \%$ glycerol buffer.

Chemical reconstitution of as-isolated $\mathrm{HydF}^{\Delta \mathrm{EG}}$ using $\mathrm{Na}_{2} \mathrm{~S}$ and $\mathrm{FeCl}_{3}$ was performed in a Coy anaerobic chamber as previously described. ${ }^{47}$ Briefly, purified HydF containing $2.24 \pm$ $0.49 \mathrm{Fe}$ atoms/dimer was supplemented with $5 \mathrm{mM}$ dithiothreitol (DTT), prior to the stepwise addition of a 6fold excess of $\mathrm{FeCl}_{3}$ and $\mathrm{Na}_{2} \mathrm{~S} \cdot 9 \mathrm{H}_{2} \mathrm{O}$. The solution was allowed to incubate for $2.5 \mathrm{~h}$ while being stirred, at which time the 
mixture was centrifuged to remove bulk FeS particulates; the clarified supernatant was then buffer exchanged into $50 \mathrm{mM}$ HEPES (pH 7.4), $0.3 \mathrm{M} \mathrm{KCl}$, and 5\% glycerol using a Sephadex G25 column.

All protein concentration values were calculated via the Bradford assay using a bovine serum albumin standard solution. The values reported herein for HydF protein concentration refer to the dimeric content of samples, whereas HydE and pyruvate formate lyase activating enzyme (PFL-AE) concentration values are reported for the monomeric content of samples. Iron quantification for all protein samples was performed using a Varian SpectrAA 220 FS flame atomic absorption spectrometer; unknowns were calculated against a 0.4-2.0 ppm Fe standard curve made from a 1000 ppm Iron AA standard (Ricca Chemical Co.).

Protein Expression and Purification of HydE and PFLAE. C-terminally His-tagged C.a. HydE was purified and chemically reconstituted according to our previously published methods. ${ }^{20}$ All HydE protein used herein contained 7.64 \pm 0.10 $\mathrm{Fe}$ atoms/protein, and all HydE samples were prepared in a 25 $\mathrm{mM}$ HEPES ( $\mathrm{pH} 8.0$ ), $0.5 \mathrm{M} \mathrm{KCl}, 5 \%$ glycerol buffer. Previously published methods were followed for the purification of PFL-AE. ${ }^{48,49}$ PFL-AE samples utilized herein contained $2.70 \pm 0.10 \mathrm{Fe}$ atoms/protein and were prepared in a $50 \mathrm{mM}$ Tris, $200 \mathrm{mM} \mathrm{NaCl}$ ( $\mathrm{pH} 7.5$ ) buffer.

Gel Filtration. Samples of C.a. HydF were analyzed via Superose 12 (GE Healthcare) size-exclusion chromatography (HR 10/30 column; $1 \mathrm{~cm}$ inside diameter, $30 \mathrm{~cm}$ length) at room temperature within a Coy anaerobic chamber, maintained as described above. Column equilibration into a $50 \mathrm{mM}$ HEPES ( $\mathrm{pH}$ 7.4), $0.3 \mathrm{M} \mathrm{KCl}, 5 \%$ glycerol buffer was accomplished using an ÄKTA Purifier FPLC instrument (GE Healthcare) at a flow rate of $0.2 \mathrm{~mL} / \mathrm{min}$. Sample runs were performed at least in duplicate on one of two columns, with slightly different bed volumes. The sample oligomeric content was calibrated against a Bio-Rad standard (\#151-1901) that contained thyroglobin (bovine), $\gamma$-globulin (bovine), ovalbumin (chicken), myoglobin (horse), and vitamin $B_{12}$. Samples were injected either into the mixer port of the FPLC with a $\sim 2$ $\mathrm{ft}$ tube $(0.076 \mathrm{~cm}$ inside diameter) lead on the column or directly onto the column. Under these conditions, tetrameric $(\sim 189 \mathrm{kDa})$ and dimeric $(\sim 94.5 \mathrm{kDa})$ HydF species eluted with retention volumes of $\sim 9-11$ and $\sim 10-12 \mathrm{~mL}$, respectively, with variability due to altered injection techniques or the specific column used. Separate calibration curves using the Bio-Rad standard solution were created for each sample injection to ensure accurate assessment of $\mathrm{HydF}$ oligomeric forms.

HydF Dimer versus Tetramer Activation of HydA ${ }^{\Delta \mathrm{EFG}}$. Gel filtration of $\mathrm{HydF}^{\mathrm{EG}}$ was linked to in vitro hydrogenase activation assays to directly probe which quaternary state of $\mathrm{HydF}$ is responsible for $\mathrm{HydA}^{\triangle \mathrm{EFG}}$ activation. Aliquots of purified $\mathrm{HydF}^{\mathrm{EG}}$ were run over the Superose $12 \mathrm{HR}$ 10/30 column, and peak fractions [as judged by the $280 \mathrm{~nm}$ (protein) and $426 \mathrm{~nm}$ (FeS LMCT band) absorbance traces] associated with tetramer and dimer states were collected and immediately incubated with $\mathrm{HydA}^{\mathrm{AEFG}}$. Assays $(2 \mathrm{~mL}$ final volume $)$ were prepared in a Coy or MBraun anaerobic chamber at room temperature in $24 \mathrm{~mL}$ sealable glass crimp vials. Reaction mixtures were supplemented with sodium dithionite and reactions initiated via addition of oxidized methyl viologen; in this scheme, dithionite acts as an electron donor for methyl viologen, which serves as an electron conduit to HydA. ${ }^{43}$ The assay concentrations were as follows: $50 \mathrm{mM}$ HEPES ( $\mathrm{pH} 7.4$ ), $300 \mathrm{mM} \mathrm{KCl}, 5 \%$ glycerol, $20 \mathrm{mM}$ dithionite, $10 \mathrm{mM}$ methyl viologen, $0.3-0.5 \mu \mathrm{M} \mathrm{HydA}{ }^{\Delta \mathrm{EFG}}$, and 3-50 $\mu \mathrm{M} \mathrm{HydF}^{\mathrm{EG}}$. HydA was purified from either Chlamydomonas reinhardtii (C.r.) (His-tagged) or Clostridium pasteurianum I (C.p.I) (streptagged) to a final concentration of $35-50 \mathrm{mg} / \mathrm{mL}$, as per previous protocols. ${ }^{14,40,43} \mathrm{HydF}^{\mathrm{EG}}$ and $\mathrm{HydA}^{\mathrm{AFF}}$ were incubated at $30{ }^{\circ} \mathrm{C}$ for $5-10 \mathrm{~min}$, prior to the addition of dithionite and methyl viologen. Assay preparation occurred in an MBraun chamber at an $\mathrm{O}_{2}$ concentration of $\leq 1 \mathrm{ppm}$ using buffers freshly deoxygenated on a Schlenk line. For the duration of the assay, the samples were kept at either 30 or $37{ }^{\circ} \mathrm{C}$ while being agitated slightly. The production of $\mathrm{H}_{2}(\mathrm{~g})$ was monitored via gas chromatography, as previously described. ${ }^{43}$

To determine the hydrogen concentration, the sample assay hydrogen content was compared to a standard calibration curve of a hydrogen/nitrogen (1:99) gas mixture. The hydrogen content was measured with a Shimadzu GC-8A gas chromatography instrument with a thermal conductivity detector, a nitrogen carrier gas, and a Supelco 80/100 Porapak $\mathrm{N}$ column $(6 \mathrm{ft} \times 1 / 8$ in. $)$ with a column temperature of $70^{\circ} \mathrm{C}$ and an injection/detector temperature of $100{ }^{\circ} \mathrm{C}$. Under these conditions, the retention time of hydrogen was $\sim 0.9 \mathrm{~min}$. For each assay time point, at least two technical replicates were taken, and the assays were performed multiple times. Assay headspace was injected on the gas chromatograph manually with a gastight Hamilton (50 or $100 \mu \mathrm{L}$ ) syringe whose gas space was purged with $100 \%$ nitrogen prior to sample headspace withdrawal.

Electron Paramagnetic Resonance Sample Preparation and Data Collection. EPR samples were all prepared in an MBraun chamber at $\mathrm{O}_{2}$ levels of $\leq 1 \mathrm{ppm}$ using buffers that had been freshly degassed on a Schlenk line. Protein samples were loaded into EPR tubes (Wilmad LabGlass, $4 \mathrm{~mm}$ outside diameter), capped with a rubber septum, and then immediately flash-frozen outside the chamber in liquid $\mathrm{N}_{2}$. Photoreduced protein was prepared by supplementing $\mathrm{HydF}$ [in $50 \mathrm{mM}$ HEPES (pH 7.4), $0.3 \mathrm{M} \mathrm{KCl}, 5 \%$ glycerol buffer] with $5 \mathrm{mM}$ DTT and $100 \mu \mathrm{M}$ 5-deazariboflavin in $50 \mathrm{mM}$ Tris ( $\mathrm{pH} 7.4$ ) buffer and illuminating samples with a $300 \mathrm{~W}$ Xe lamp in an ice/water bath for $1 \mathrm{~h}$. Illumination of target protein samples in the presence of 5-deazariboflavin and Tris buffer (the source of reducing equivalents) produces a catalytic source of lowpotential electrons. ${ }^{50}$ Immediately following photoillumination, EPR samples were flash-frozen and stored under liquid $\mathrm{N}_{2}$ until data were collected.

Low-temperature $(\leq 70 \mathrm{~K})$ continuous wave $(\mathrm{CW})$, X-band EPR measurements at Montana State University were taken using a Bruker EMX spectrometer fitted with a Bruker/Cold Edge (Sumitomo Cryogenics) $10 \mathrm{~K}$ waveguide cryogen-free cooling system and an Oxford MercuryiTC controller unit. The helium gas flow was maintained at $100 \mathrm{psi}$, and unless otherwise indicated, the set point for the sample temperature setting was $10.5 \mathrm{~K}$. Typical EPR parameters were as follows: $9.38 \mathrm{GHz}$ microwave frequency, $100 \mathrm{kHz}$ modulation frequency, $3 \mathrm{G}$ modulation amplitude, $163.84 \mathrm{~ms}$ time constant, and spectra averaged over four scans. OriginPro version 8.5 (OriginLab Corp., Northampton, MA) was used to baseline correct and plot all experimental spectra.

$\mathrm{X}$-Band CW and pulsed EPR data were acquired on a Bruker E580 spectrometer at the University of Denver using a split ring resonator and an Oxford ESR935 cryostat. Spin-spin relaxation times, $T_{2}$, were measured by two-pulse spin echo using a $90^{\circ}-$ 
$180^{\circ}$ pulse sequence and a $90^{\circ}$ pulse length of 40 ns. Spinlattice relaxation was measured by inversion recovery using a $180^{\circ}-90^{\circ}-180^{\circ}$ pulse sequence and a $90^{\circ}$ pulse length of 40 ns. For the $[2 \mathrm{Fe}-2 \mathrm{~S}]^{+}$cluster, measurements were performed at a $g_{\perp}$ value of 2.006 and at an intermediate $g$ value of 1.99 , which is higher than the $g_{\|}$value of 1.96. There does not appear to be significant anisotropy in $T_{1}$. The spin echo decays and inversion recovery curves were fit with a single exponential, unless otherwise noted. Standard deviations for replicate measurements are $\sim 5 \%$.

The $g$ values and inhomogeneous broadening of the lowtemperature $\mathrm{CW}$ spectra were found by simulation using the locally written program MONMER that is based on the equations in ref 51 . The $g_{\perp}$ regions of the temperaturedependent $\mathrm{CW}$ spectra at $110-150 \mathrm{~K}$ were simulated using SATMON in which the line shape is a Gaussian distribution of Lorentzian spin packets characterized by $T_{2} .{ }^{52}$ In the temperature range in which line widths are temperaturedependent, it was assumed that $T_{1}=T_{2}$ for $[2 \mathrm{Fe}-2 \mathrm{~S}]^{+}$. A detailed description of the calculations is given in ref 53 . The temperature dependence of $T_{1}$ for $[2 \mathrm{Fe}-2 \mathrm{~S}]^{+}$was fit with the function $^{53}$

$$
\frac{1}{T_{1}}=C_{\text {dir }}+C_{\text {Ram }}\left(\frac{T}{\theta_{\mathrm{D}}}\right)^{9} J_{8}\left(\frac{\theta_{\mathrm{D}}}{T}\right)+C_{\mathrm{orb}} \frac{\Delta_{\mathrm{orb}}{ }^{3}}{\mathrm{e}^{\Delta_{\mathrm{orb} / T}}-1}
$$

where $T$ is the temperature in kelvin, $C_{\mathrm{dir}}$ is the contribution from the direct process, $C_{\text {Ram }}$ is the coefficient for the contribution from the Raman process, $\theta_{\mathrm{D}}$ is the Debye temperature, $J_{8}$ is the transport integral, $J_{8}\left(\frac{\theta_{\mathrm{D}}}{T}\right)=\int_{0}^{\theta_{\mathrm{D}} / T} x^{8} \frac{\mathrm{e}^{x}}{\left(\mathrm{e}^{x}-1\right)^{2}} \mathrm{~d} x, C_{\text {orb }}$ is the coefficient for the contribution from the Orbach process, and $\Delta_{\text {orb }}$ is the energy separation between the ground and excited states for the Orbach process.

\section{RESULTS}

The capacity to achieve HydA ${ }^{\Delta \mathrm{EFG}}$ activation by $\mathrm{HydF}^{\mathrm{EG}}$ in the absence of any exogenous small molecules or accessory proteins supports the notion that $\mathrm{HydF}$ either acts as a carrier of, or serves as a scaffold for, assembly of the $2 \mathrm{Fe}$ subcluster of the $\mathrm{H}$ cluster. ${ }^{14,43,54,55}$ Assembly of the $2 \mathrm{Fe}$ subcluster on $\mathrm{HydF}^{\mathrm{EG}}$ is additionally supported by the spectroscopic observation of FTIR bands associated with $\mathrm{Fe}-\mathrm{CO}$ and $\mathrm{Fe}-\mathrm{CN}^{-}$species in purified protein, as well as XAS results that point to both a dinuclear iron unit and a [4Fe-4S] cluster. ${ }^{40,45,56}$ Until recently, there were some discrepancies in the literature regarding the nature of $\mathrm{FeS}$ clusters coordinated by HydF prior to its loading by HydE and HydG. Defining the FeS cluster species bound to $\mathrm{HydF}^{\mathrm{AEG}}$ is essential for understanding how this protein acts as either a scaffold or a carrier during maturation, as it clarifies the nature of the chemical species that HydE and HydG deliver to HydF. A comprehensive spectroscopic study using UV-vis, $\mathrm{CD}$, and EPR techniques on the effects of sample handling on HydF demonstrated that $\mathrm{HydF}^{\mathrm{EEG}}$ coordinates both redoxactive $[4 \mathrm{Fe}-4 \mathrm{~S}]^{2+/+}$ and $[2 \mathrm{Fe}-2 \mathrm{~S}]^{2+/+}$ clusters. $^{47}$ Outstanding questions remain, however, including whether the $[4 \mathrm{Fe}-4 \mathrm{~S}]$ and [2Fe-2S] clusters are bound simultaneously to a single protein subunit and if these clusters are bound in the proximity of each other. Here we describe pulsed EPR spectroscopy and relaxation enhancement calculations, together with biochemical studies, that aim to address these questions.
EPR Spectroscopy. Previously, we have shown that treatment of as-purified $\mathrm{HydF}^{\mathrm{EG}}$ with DTT causes reduction of existing $[2 \mathrm{Fe}-2 \mathrm{~S}]^{2+}$ states and intensification of $[2 \mathrm{Fe}-2 \mathrm{~S}]^{+}$ cluster EPR signals; preparation of DTT-treated $\mathrm{HydF}^{\Delta \mathrm{EG}}$ thus results in an enzyme that exhibits a $[2 \mathrm{Fe}-2 \mathrm{~S}]^{+}$cluster without any other overlapping $\mathrm{FeS}$ cluster signals (Figure 3A). ${ }^{47}$ In
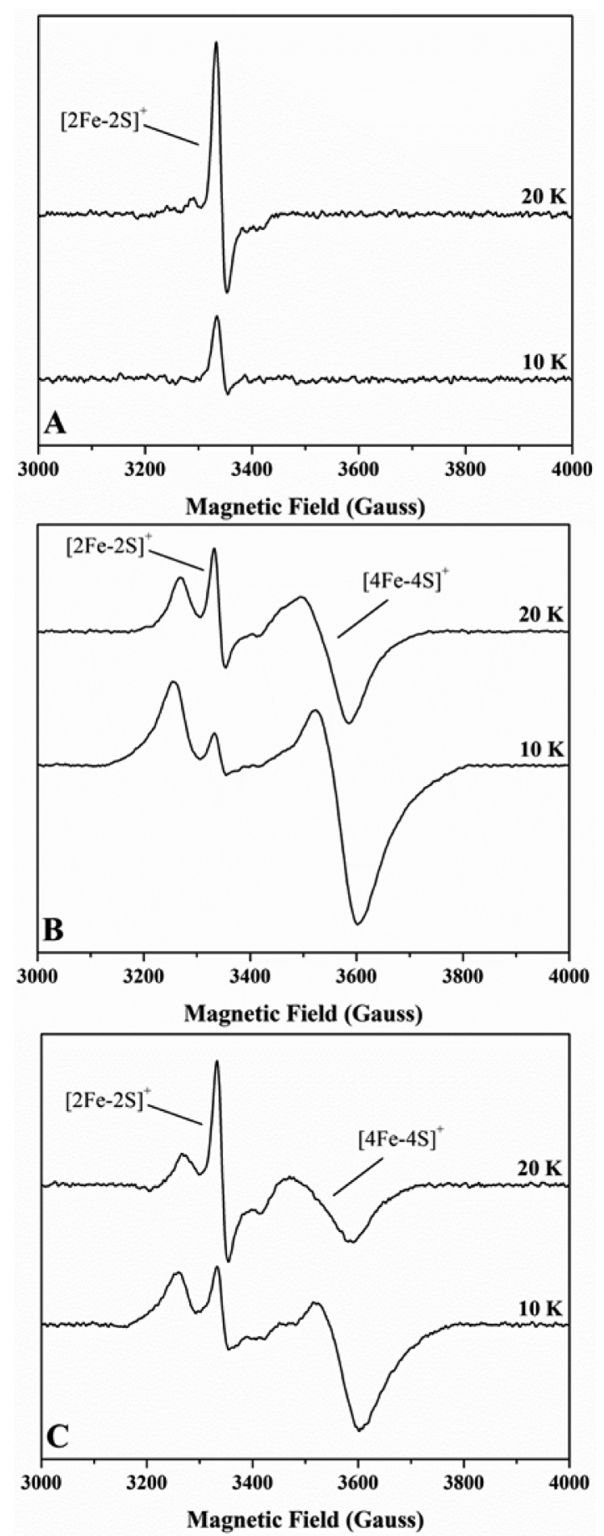

Figure 3. HydF low-temperature X-band CW EPR spectra for samples studied by pulsed EPR. (A) Freshly purified $\mathrm{HydF}^{\Delta \mathrm{EG}}(104 \mu \mathrm{M}$ protein at $2.2 \pm 0.1 \mathrm{Fe}$ atoms/dimer) in the presence of $5 \mathrm{mM}$ dithiothreitol. (B) As-isolated $\mathrm{HydF}^{\Delta \mathrm{EG}}(600 \mu \mathrm{M}$ protein at $1.1 \pm 0.1$ Fe atoms/dimer) following photoreduction. (C) Chemically reconstituted $\mathrm{HydF}^{\Delta \mathrm{EG}}(78 \mu \mathrm{M}$ protein at $5.0 \pm 0.7 \mathrm{Fe}$ atoms/dimer $)$ following photoreduction. Spectra were all recorded at a microwave power of $800 \mu \mathrm{W}$ with the same gain settings.

contrast, as-isolated and chemically reconstituted $\mathrm{HydF}^{\Delta \mathrm{EG}}$ samples that are photoreduced in the presence of DTT are poised in a state in which both $[2 \mathrm{Fe}-2 \mathrm{~S}]^{+}$and $[4 \mathrm{Fe}-4 \mathrm{~S}]^{+}$cluster EPR signals are observed (Figure $3 \mathrm{~B}, \mathrm{C}){ }^{47}$ EPR spectroscopy was used to provide insight into the electronic structure and local environment of these clusters and to determine the electron spin relaxation properties of $\mathrm{HydF}^{\Delta \mathrm{EG}}$ containing these 
two different FeS cluster species, which allowed us to examine the spatial proximity of the $[2 \mathrm{Fe}-2 \mathrm{~S}]^{+}$cluster to the $[4 \mathrm{Fe}-4 \mathrm{~S}]^{+}$ cluster. $^{57-59}$

We also performed the same spectroscopic measurements using PFL-AE and HydE, to provide controls to support our interpretation of the HydF results. PFL-AE is a member of the radical SAM superfamily and harbors a single $\mathrm{FeS}$ cluster binding $\left(\mathrm{CX}_{3} \mathrm{CX}_{2} \mathrm{C}\right)$ motif. $^{19}$ As-purified PFL-AE coordinates predominantly $[4 \mathrm{Fe}-4 \mathrm{~S}]^{2+}$ and $[3 \mathrm{Fe}-4 \mathrm{~S}]^{+}$clusters at this site, although some preparations additionally contain small amounts of $[2 \mathrm{Fe}-2 \mathrm{~S}]^{+}$clusters (Figure S1); in all cases, however, a PFL$\mathrm{AE}$ molecule can bind only a single cluster, so this protein serves as a standard for cluster spin relaxation that is unperturbed by a nearby cluster. ${ }^{47,48,60-62} \mathrm{HydE}$ has two iron-sulfur cluster binding motifs, with an accessory Cys311, Cys319, and Cys322 FeS cluster binding site located approximately $20 \AA$ from the radical SAM $\mathrm{CX}_{3} \mathrm{CX}_{2} \mathrm{C}$ motif. ${ }^{63}$ Structural studies of T.m. HydE have shown that the accessory site either can be vacant or can coordinate a $[2 \mathrm{Fe}-2 \mathrm{~S}]$ or $[4 \mathrm{Fe}-$ $4 \mathrm{~S}$ ] cluster, depending on preparation conditions. ${ }^{63,64} \mathrm{We}$ showed that in some preparations of as-reconstituted C.a. HydE this site was occupied by a $[2 \mathrm{Fe}-2 \mathrm{~S}]^{+}$cluster, based on the iron number following chemical reconstitution and EPR spectra as a function of temperature that confirm the presence of both $[3 \mathrm{Fe}-4 \mathrm{~S}]^{+}$(presumably coordinated to the $\mathrm{CX}_{3} \mathrm{CX}_{2} \mathrm{C}$ motif) and $[2 \mathrm{Fe}-2 \mathrm{~S}]^{+}$cluster signals (Figure S2). ${ }^{20,47} \mathrm{HydE}$ therefore provided a means for us to examine $[2 \mathrm{Fe}-2 \mathrm{~S}]^{+}$cluster spin relaxation in a sample that contains a fast-relaxing $[3 \mathrm{Fe}-4 \mathrm{~S}]^{+}$ cluster coordinated $\approx 20 \AA$ away (see below).

The dominant contributions to the spin relaxation for both $[2 \mathrm{Fe}-2 \mathrm{~S}]^{+}$and $[4 \mathrm{Fe}-4 \mathrm{~S}]^{+}$clusters are the Raman and Orbach processes. $^{57-59,65-67}$ The spin relaxation rates that result from both processes are determined by the spin-orbit coupling, where larger coupling enhances relaxation. ${ }^{58,68}$ The Orbach energy, $\Delta_{\text {orb }}$, is the energy separation between the ground state and the lowest excited state, which is determined by the spinspin interaction within the iron-sulfur cluster. Literature values of $\Delta_{\text {orb }}$ obtained by analysis of the temperature dependence of electron spin-lattice relaxation are $250-570 \mathrm{~cm}^{-1}$ for $[2 \mathrm{Fe}$ $2 \mathrm{~S}]^{+}$clusters, ${ }^{57,58,69} 88 \mathrm{~cm}^{-1}$ for a $[3 \mathrm{Fe}-4 \mathrm{~S}]^{+}$ferredoxin cluster, $^{70}$ and $120-140 \mathrm{~cm}^{-1}$ for two $[4 \mathrm{Fe}-4 \mathrm{~S}]^{+}$clusters. $^{59,66}$ Literature values of the Debye temperature (eq 1) are 60-120 $\mathrm{K}$ for $[2 \mathrm{Fe}-2 \mathrm{~S}]^{+}$clusters $^{58,65}$ and $60-100 \mathrm{~K}$ for $[4 \mathrm{Fe}-4 \mathrm{~S}]^{+}$ clusters. $^{59,66}$

The CW spectra of the reduced $\mathrm{HydF}^{\Delta \mathrm{EG}}$ samples between 10 and $70 \mathrm{~K}$ (Figure 3) are similar to spectra shown in the Supporting Information of previous work. ${ }^{40}$ At $<100 \mathrm{~K}$, the line widths of the $\mathrm{CW}$ spectra of the $[2 \mathrm{Fe}-2 \mathrm{~S}]^{+}$cluster are independent of temperature and are attributed to $g$ anisotropy, distributions in $g$ values, and unresolved nuclear hyperfine interactions. The temperature independence of the line widths indicates they are not dominated by electron spin relaxation. At $>110 \mathrm{~K}$, the signal from the $[2 \mathrm{Fe}-2 \mathrm{~S}]^{+}$cluster in $\mathrm{HydF}^{\Delta \mathrm{EG}}$ is broadened significantly (Figure S3B); this behavior for the C.a. $\mathrm{HydF}^{\mathrm{EEG}}[2 \mathrm{Fe}-2 \mathrm{~S}]^{+}$cluster signal is parallel to that reported for as-purified S.o. $\mathrm{HydF}^{\Delta \mathrm{EG}}{ }^{71}$ In this temperature regime, $T_{2}$ becomes short enough that the relaxation broadening of the signal is significant relative to the inhomogeneous broadening that defines the line widths at lower temperatures. For PFL-AE and $\mathrm{HydF}^{\Delta \mathrm{EG}}$ samples, spectra at $80 \mathrm{~K}$ were simulated with SATMON $^{52}$ to evaluate the $g$ values and the non-relaxationdependent contributions to the line widths. Those parameters were held constant, and the spin relaxation rates $\left(1 / T_{2}\right)$ were varied to fit the spectra at $110-150 \mathrm{~K}$ (Figure S3). Analysis of the temperature-dependent contribution to the line widths and the assumption that $T_{1} \sim T_{2}$, in the line-broadening regime, can be used to calculate $T_{1}$. Importantly, the temperaturedependent broadening at $110-150 \mathrm{~K}$ is similar for the [2Fe$2 \mathrm{~S}^{+}$signals in PFL-AE, photoreduced $\mathrm{HydF}^{\Delta \mathrm{EG}}$, and freshly purified $\mathrm{HydF}^{\Delta \mathrm{EG}}$ treated with DTT. Because the spin-lattice relaxation rates for $[4 \mathrm{Fe}-4 \mathrm{~S}]^{+}$and $[3 \mathrm{Fe}-4 \mathrm{~S}]^{+}$clusters are much faster than for the $[2 \mathrm{Fe}-2 \mathrm{~S}]^{+}$cluster, the signals for the $[4 \mathrm{Fe}-$ $4 \mathrm{~S}]^{+}$or $[3 \mathrm{Fe}-4 \mathrm{~S}]^{+}$clusters are observed in the CW spectra only at less than $\sim 50 \mathrm{~K},{ }^{40}$ and less than $\sim 25 \mathrm{~K}$ in the field-swept echo-detected spectra (Figure S4).

Direct measurements of $T_{1}$ and $T_{2}$ for the $[2 \mathrm{Fe}-2 \mathrm{~S}]^{+}$cluster in $\mathrm{HydF}^{\Delta \mathrm{EG}}$ and related samples were obtained by inversion recovery and spin echo decay below $\approx 60 \mathrm{~K}$. Below $20 \mathrm{~K}$, overlap with the much broader signals from the $[4 \mathrm{Fe}-4 \mathrm{~S}]^{+}$and $[3 \mathrm{Fe}-4 \mathrm{~S}]^{+}$clusters made it difficult to distinguish contributions to the inversion recovery curves. The temperature dependence of $T_{2}$ measured by spin echo is shown in Figure 4. Values of $T_{2}$

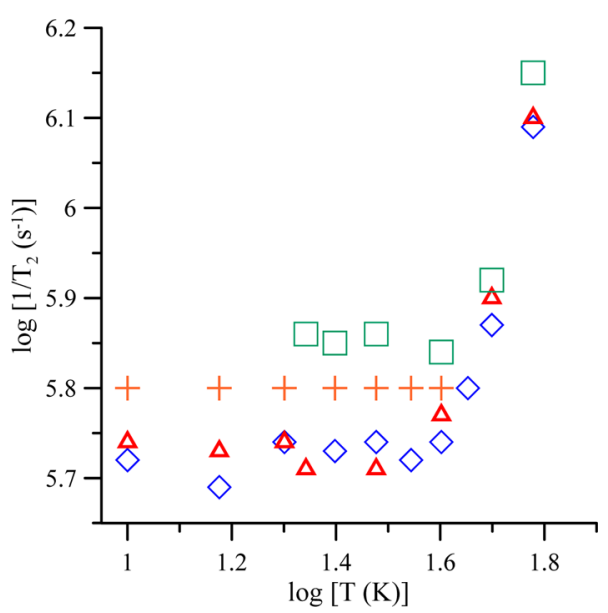

Figure 4. Temperature dependence of X-band spin-spin relaxation rates at $g=2.006$ for the $[2 \mathrm{Fe}-2 \mathrm{~S}]^{+}$cluster signals in various samples. Data are shown for the following proteins: as-purified PFL-AE [1.68 $\mathrm{mM}$ protein with $2.7 \pm 0.1 \mathrm{Fe}$ atoms/protein (green squares)], asreconstituted $\mathrm{HydE}[340 \mu \mathrm{M}$ protein with $7.6 \pm 0.1 \mathrm{Fe}$ atoms/protein (orange pluses)], as-isolated, photoreduced $\mathrm{HydF}^{\Delta \mathrm{EG}}[600 \mu \mathrm{M}$ protein at $1.1 \pm 0.1 \mathrm{Fe}$ atoms/dimer (red triangles)], and freshly purified $\mathrm{HydF}^{\Delta \mathrm{EG}}(104 \mu \mathrm{M}$ protein at $2.2 \pm 0.1 \mathrm{Fe}$ atoms/dimer $)$ in the presence of $5 \mathrm{mM}$ dithiothreitol (blue diamonds).

below $\sim 40 \mathrm{~K}$ are approximately independent of temperature, and $T_{2}$ is between about 1.5 and $1.9 \mu \mathrm{s}$, which is in the range that is found for many $S=1 / 2$ species at relatively low spin concentrations. ${ }^{53}$ The temperature independence below $40 \mathrm{~K}$ suggests that the spin-spin relaxation is dominated by nuclear spin diffusion among the many proton spins in the vicinity of the iron-sulfur clusters. Above $\sim 40 \mathrm{~K}$, the values of $T_{2}$ become strongly temperature dependent because of the increasingly fast spin-lattice relaxation and its contribution to $T_{2}$. It should be noted that the data shown in Figure 4 for photoreduced $\mathrm{HydF}^{\Delta \mathrm{EG}}$ are for the as-isolated photoreduced enzyme. Importantly, the values for as-isolated, photoreduced $\mathrm{HydF}^{\Delta \mathrm{EG}}$ are indistinguishable from data obtained for chemically reconstituted, photoreduced $\mathrm{HydF}^{\Delta \mathrm{EG}}$ that were recorded at a subset of these temperatures (data not shown).

The temperature dependence of $T_{1}$ for the $[2 \mathrm{Fe}-2 \mathrm{~S}]^{+}$cluster in $\mathrm{HydF}^{\Delta \mathrm{EG}}$ and related samples is summarized in Figure 5. 

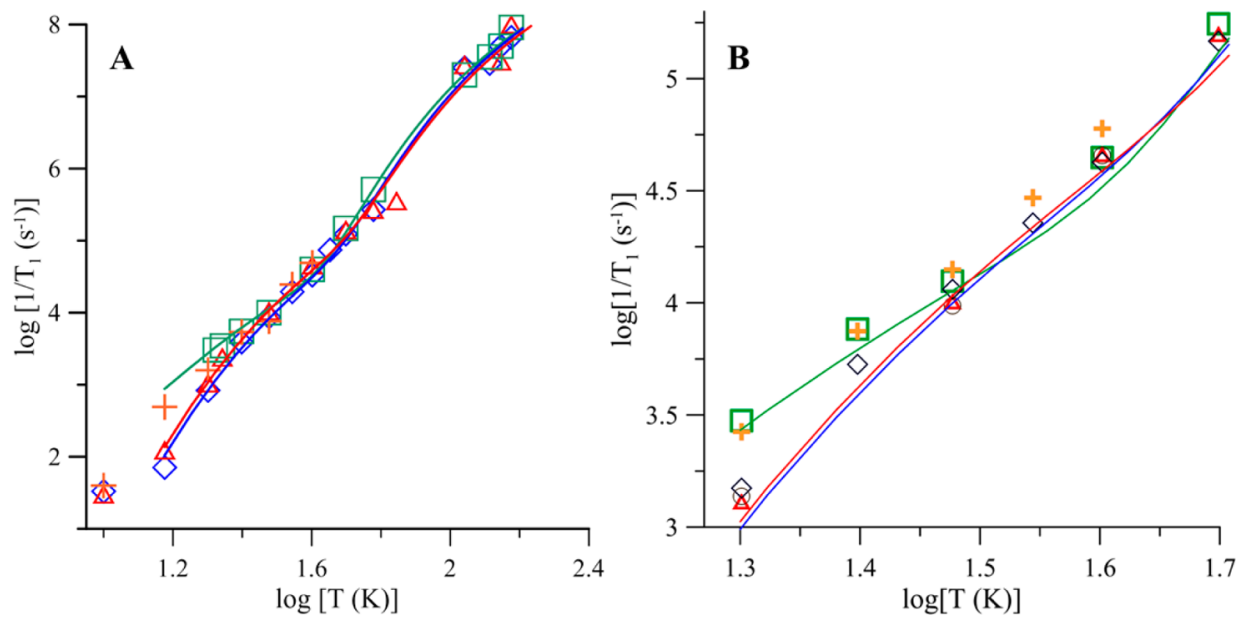

Figure 5. Temperature dependence of X-band spin-lattice relaxation rates at $g=2.006$ for the $[2 \mathrm{Fe}-2 \mathrm{~S}]^{+}$cluster signals in various samples. (A) The data are for the following samples: as-purified PFL-AE [1.68 mM protein with $2.7 \pm 0.1 \mathrm{Fe}$ atoms/protein (green squares)], as-reconstituted HydE $[340 \mu \mathrm{M}$ protein with $7.6 \pm 0.1 \mathrm{Fe}$ atoms/protein (orange pluses) $]$, and freshly purified $\mathrm{HydF}^{\Delta \mathrm{EG}}(104 \mu \mathrm{M}$ protein at $2.2 \pm 0.1 \mathrm{Fe}$ atoms/dimer) in the presence of $5 \mathrm{mM}$ dithiothreitol (blue diamonds). The red triangles represent a merged data set for measurements collected on both as-isolated, photoreduced $\mathrm{HydF}^{\Delta \mathrm{EG}}\left(600 \mu \mathrm{M}\right.$ protein at $1.1 \pm 0.1 \mathrm{Fe}$ atoms/dimer) and chemically reconstituted, photoreduced $\mathrm{HydF}^{\Delta \mathrm{EG}}(78 \mu \mathrm{M}$ protein at 5.0 $\pm 0.7 \mathrm{Fe}$ atoms/dimer). Data were obtained for as-isolated, photoreduced $\mathrm{HydF}^{\Delta \mathrm{EG}}$ and chemically reconstituted, photoreduced $\mathrm{HydF}^{\Delta \mathrm{EG}}$ samples between 17 and $40 \mathrm{~K}$. Data for chemically reconstituted, photoreduced $\mathrm{HydF}^{\Delta \mathrm{EG}}$ were analyzed at $110-150 \mathrm{~K}$. The solid lines are the modeling of the relaxation rates for PFL-AE (green), the merged data set for photoreduced $\mathrm{HydF}^{\Delta \mathrm{EG}}$ (red), and that for freshly purified $\mathrm{HydF}^{\Delta \mathrm{EG}}$ in the presence of $5 \mathrm{mM}$ dithiothreitol (blue) as the sum of contributions from the Raman and Orbach processes. (B) Expanded plot of data between $20 \mathrm{~K}$ (log $T=$ $1.3)$ and $50 \mathrm{~K}(\log T=1.7)$. The symbols and color designations are the same as in panel A except that data for as-isolated, photoreduced HydF ${ }^{\Delta \mathrm{EG}}$ are represented by the red triangles and data for chemically reconstituted, photoreduced $\mathrm{HydF}^{\Delta \mathrm{EG}}$ are represented by brown circles.

Table 1. Parameters for Modeling the Temperature Dependence of $1 / T_{1}$ for the $[2 \mathrm{Fe}-2 \mathrm{~S}]^{+}$Cluster Signals

\begin{tabular}{lccccc}
\multicolumn{1}{c}{ sample } & $A_{\text {dir }}\left(\mathrm{s}^{-1}\right)$ & $\theta_{\mathrm{D}}(\mathrm{K})$ & $C_{\mathrm{Ram}}\left(\mathrm{s}^{-1} \mathrm{~K}^{-9}\right)$ & $\Delta_{\text {orb }}(\mathrm{K})$ & $C_{\text {orb }}\left(\mathrm{s}^{-1} \mathrm{~K}^{-3}\right)$ \\
PFL-AE & $16 \pm 2$ & $100 \pm 20$ & $(1.6 \pm 0.4) \times 10^{6}$ & $500 \pm 50$ & $15 \pm 3$ \\
photoreduced $\mathrm{HydF}^{\mathrm{AEG} a}$ & $0^{b}$ & $170 \pm 20$ & $(13 \pm 3) \times 10^{6}$ & $560 \pm 50$ & $14 \pm 3$ \\
freshly purified HydF ${ }^{\Delta \mathrm{EG}}$ with $5 \mathrm{mM} \mathrm{DTT}$ & $0^{b}$ & $170 \pm 20$ & $(10 \pm 3) \times 10^{6}$ & $550 \pm 50$ & $15 \pm 3$
\end{tabular}

${ }^{a}$ Values are reported for the merged data set created by measurements with both as-isolated, photoreduced HydF ${ }^{\Delta \mathrm{EG}}$ and chemically reconstituted, photoreduced $\mathrm{HydF}^{\Delta \mathrm{EG}}$ samples (see the legend of Figure 5). ${ }^{b}$ Values of $T_{1}$ at $10 \mathrm{~K}$ were not included in the modeling. The limited data at temperatures of $<20 \mathrm{~K}$ were insufficient to define $A_{\text {dir }}$.

Values of $T_{1}$ below $\approx 60 \mathrm{~K}$ were obtained by inversion recovery, and values at temperatures between 110 and $150 \mathrm{~K}$ were obtained by analysis of the temperature-dependent contributions to the CW line shapes (see above and Figure S3). $T_{1}$ relaxation times for the $[2 \mathrm{Fe}-2 \mathrm{~S}]^{+}$cluster signals in as-isolated, photoreduced $\mathrm{HydF}^{\Delta \mathrm{EG}}$ and in chemically reconstituted, photoreduced $\mathrm{HydF}^{\Delta \mathrm{EG}}$ were so similar that the results are treated as a combined data set in Figure 5 (see the legend for details). The relaxation rates for these clusters are more than an order of magnitude slower than those reported previously for other $[2 \mathrm{Fe}-2 \mathrm{~S}]^{+}$clusters. ${ }^{58}$ The relaxation rates for PFL-AE, photoreduced $\mathrm{HydF}^{\Delta \mathrm{EG}}$, and freshly purified $\mathrm{HydF}^{\Delta \mathrm{EG}}$ reduced with DTT were modeled as the sum of contributions from the Raman and Orbach processes. There is substantial uncertainty in the Debye temperatures because there is a relatively narrow temperature range (approximately $15-35 \mathrm{~K}$ ) in which the Raman process dominates. However, the significantly smaller value of $\theta_{\mathrm{D}}$ for the $[2 \mathrm{Fe}-2 \mathrm{~S}]^{+}$cluster signal in PFL-AE, relative to that of $\mathrm{HydF}^{\mathrm{AEG}}$, suggests substantially different local environments for the $[2 \mathrm{Fe}-2 \mathrm{~S}]^{+}$clusters between these samples (Table 1). The Orbach process dominates at higher temperatures. The Orbach energies for the $[2 \mathrm{Fe}-2 \mathrm{~S}]^{+}$clusters in PFL$\mathrm{AE}$ and in $\mathrm{HydF}^{\Delta \mathrm{EG}}$ range between 500 and $560 \mathrm{~K}$ (Table 1, $350 \pm 35 \mathrm{~cm}^{-1}$ ), which is approximately in the middle of the range for previously reported values. ${ }^{57,58,69}$ The coefficients for the Raman process, $C_{\text {Ram }}$ that were used to generate the fit lines shown in Figure 5 are $10^{6}-10^{7} \mathrm{~s}^{-1} \mathrm{~K}^{-9}$ (Table 1), which are several orders of magnitude smaller than the value of $\sim 10^{10}$ $\mathrm{s}^{-1} \mathrm{~K}^{-9}$ reported for other $[2 \mathrm{Fe}-2 \mathrm{~S}]^{+}$clusters. ${ }^{58}$ Similarly, the values of $C_{\text {orb }}$ for $\mathrm{HydF}^{\Delta \mathrm{EG}}$ are $\sim 15 \mathrm{~s}^{-1} \mathrm{~K}^{-3}$ (Table 1), which is more than an order of magnitude smaller than the value of $\sim 10^{3} \mathrm{~s}^{-1} \mathrm{~K}^{-3}$ reported for other $[2 \mathrm{Fe}-2 \mathrm{~S}]^{+}$clusters. ${ }^{58}$ The smaller coefficients are required in the fitting because the rates are so much slower. The $g$ value anisotropy for the $[2 \mathrm{Fe}-2 \mathrm{~S}]^{+}$ cluster signal in $\mathrm{HydF}^{\Delta \mathrm{EG}}$ is only 2.006-1.96 $=0.046$, which is considerably smaller than those reported for other $[2 \mathrm{Fe}-2 \mathrm{~S}]^{+}$ cluster signals, such as the Rieske cluster from cytochrome $b c_{1}$ $(2.0265-1.7670=0.2595) .{ }^{58}$ Smaller $g$ anisotropy indicates smaller spin-orbit coupling, which leads to slower electron spin relaxation. ${ }^{58,68}$ It has also been proposed that the coefficients for the Raman and Orbach processes are smaller for more rigid systems. ${ }^{58,72}$ The very small values of $C_{\mathrm{Ram}}$ and $C_{\text {orb }}$ for the $[2 \mathrm{Fe}-2 \mathrm{~S}]^{+}$cluster in HydF ${ }^{\Delta \mathrm{EG}}$ and PFL-AE (Table 1) suggest that the environment of the cluster in these systems is relatively rigid.

Relaxation Enhancement Calculations: Distance Constraints on $\mathrm{HydF}^{\Delta \mathrm{EG}}[2 \mathrm{Fe}-2 \mathrm{~S}]^{+}$and $[4 \mathrm{Fe}-4 \mathrm{~S}]^{+}$Cluster Signals. The plot of $\log \left(1 / T_{1}\right)$ versus $\log T$ for the $[2 \mathrm{Fe}-2 \mathrm{~S}]^{+}$ signals in various samples over the full range of temperatures studied shows that there is not a large relaxation enhancement 
(Figure 5). However, on the basis of this plot, it is difficult to evaluate subtle differences between samples. To more carefully examine small differences, Figure 5B depicts data only between 20 and $50 \mathrm{~K}$. This temperature range was selected because at less than $\sim 20 \mathrm{~K}$ the overlap with signals with more rapid relaxation rates results in inversion recovery curves with contributions from both $[2 \mathrm{Fe}-2 \mathrm{~S}]^{+}$and either $[4 \mathrm{Fe}-4 \mathrm{~S}]^{+}$or $[3 \mathrm{Fe}-4 \mathrm{~S}]^{+}$cluster signals. Above $\sim 50 \mathrm{~K}$, the signal-to-noise ratio in the inversion recovery curves is poorer and the intrinsic relaxation rates for the $[2 \mathrm{Fe}-2 \mathrm{~S}]^{+}$centers are sufficiently fast that the fractional change due to interaction with the fasterrelaxing $[4 \mathrm{Fe}-4 \mathrm{~S}]^{+}$cluster at relatively long interspin distances is smaller.

Data in Figure $5 \mathrm{~B}$ show that at $20 \mathrm{~K}$ the relaxation rates fall into two sets. The rates are very similar for the $[2 \mathrm{Fe}-2 \mathrm{~S}]^{+}$ clusters in PFL-AE and HydE; these rates are $\sim 2$-fold faster than for the $[2 \mathrm{Fe}-2 \mathrm{~S}]^{+}$cluster in $\mathrm{HydF}^{\Delta \mathrm{EG}}$ samples at $20 \mathrm{~K}$, which indicates that there are differences in the environments of the clusters between these enzymes. However, at 30-50 K, the relaxation rates for the $[2 \mathrm{Fe}-2 \mathrm{~S}]^{+}$cluster in PFL-AE are very similar to those for the $[2 \mathrm{Fe}-2 \mathrm{~S}]^{+}$cluster in $\mathrm{HydF}^{\mathrm{EEG}}$ samples. These differences are small enough that it seems reasonable to use the relaxation rates from the PFL-AE sample as models for the relaxation in $\mathrm{HydF}^{\Delta \mathrm{EG}}$ in the absence of interactions with a more rapidly relaxing paramagnetic center.

At $20-50 \mathrm{~K}$, the relaxation rates for the $[2 \mathrm{Fe}-2 \mathrm{~S}]^{+}$cluster signals in the three $\mathrm{HydF}^{\Delta \mathrm{EG}}$ samples are very similar to each other and comparable to that for the $[2 \mathrm{Fe}-2 \mathrm{~S}]^{+}$cluster in PFLAE. The notable similarity in relaxation rates between these enzymes, along with the analysis of two photoreduced $\mathrm{HydF}^{\Delta \mathrm{EG}}$ samples with different degrees of FeS cluster loading (Figure 5B), strongly argues against a significant relaxation enhancement in $\mathrm{HydF}^{\Delta \mathrm{EG}}$. The differences in relaxation rates at $20 \mathrm{~K}$ may raise some concern about the use of PFL-AE as a model system for $\mathrm{HydF}^{\mathrm{EEG}}$ (Figure $5 \mathrm{~B}$ ). However, even if the intrinsic relaxation rates in $\mathrm{HydF}^{\triangle \mathrm{EG}}$ are somewhat slower than for PFL$\mathrm{AE}$, relaxation enhancement in $\mathrm{HydF}^{\Delta \mathrm{EG}}$ by a nearby $[4 \mathrm{Fe}-4 \mathrm{~S}]^{+}$ cluster must be very small.

Importantly, at $30-40 \mathrm{~K}$, the relaxation rates for the $[2 \mathrm{Fe}$ $2 \mathrm{~S}^{+}$cluster in HydE are faster than in PFL-AE (Figure 5B), which suggests a small but significant enhancement of the relaxation rate for the $[2 \mathrm{Fe}-2 \mathrm{~S}]^{+}$cluster in HydE due to interaction with the neighboring $[3 \mathrm{Fe}-4 \mathrm{~S}]^{+}$cluster. The relaxation rate for the $[3 \mathrm{Fe}-4 \mathrm{~S}]^{+}$cluster in $\mathrm{HydE}$ is faster than for the $[2 \mathrm{Fe}-2 \mathrm{~S}]^{+}$cluster at $20 \mathrm{~K}$, but not fast enough to significantly enhance relaxation, so there is no observable relaxation enhancement for the $[2 \mathrm{Fe}-2 \mathrm{~S}]^{+}$cluster in HydE relative to that in PFL-AE at $20 \mathrm{~K}$. At $40 \mathrm{~K}$, the relaxation rate for the $[2 \mathrm{Fe}-2 \mathrm{~S}]^{+}$cluster in HydE is $\sim 35 \%$ faster than in PFL$\mathrm{AE}$, which is well beyond the estimated uncertainties in relaxation rates. The relaxation enhancement at $40 \mathrm{~K}$ is larger than at $30 \mathrm{~K}$ because of the faster relaxation rates for the $[3 \mathrm{Fe}$ $4 \mathrm{~S}]^{+}$cluster at higher temperatures. Above $\sim 40 \mathrm{~K}$, the intrinsic relaxation rate for the $[2 \mathrm{Fe}-2 \mathrm{~S}]^{+}$cluster increases so much that it becomes less sensitive to enhancement by other paramagnetic centers.

Modeling of the relaxation enhancement was performed via the program MENOSR, which has been used previously to calculate spin-lattice relaxation enhancement for interaction of paramagnetic metal centers with nitroxide radicals and semiquinone radicals. ${ }^{73-75}$ Because the two FeS clusters in HydE each have net $S=1 / 2$, this program also can be applied to mapping the cluster-cluster interaction. Figure S5 shows the inversion recovery curve at $g_{\perp}$ for the $[2 \mathrm{Fe}-2 \mathrm{~S}]^{+}$cluster signal in $\mathrm{HydE}$ at $40 \mathrm{~K}$. Simulations based on an interspin distance of 45 $\AA$ (which is defined as providing no relaxation enhancement) do not adequately fit the experimental data. However, a simulated curve using an interspin distance of $22 \AA$ does provide a relatively good fit, and thus, we are able to conclude that the interspin distance in HydE between these paramagnetic centers is $\sim 22 \AA$ (see the supplementary text associated with Figure S5). This distance should be a reasonable approximation of the distance between the centers of the two FeS clusters if we assume that the unpaired electron is uniformly distributed over the clusters. It should be noted that a distance of $22 \AA$ is consistent with the HydE X-ray crystal structure (PDB entry 3IIZ), where the distance between the centers of the two FeS clusters is $\approx 22.3 \AA .^{63}$ Calculations with MENOSR indicate that increasing the interspin distance to $\sim 25 \AA$ decreases the relaxation enhancement to the extent that it is not detectable for the $[2 \mathrm{Fe}-2 \mathrm{~S}]^{+}$cluster in $\mathrm{HydE}$ at $30-40 \mathrm{~K}$ (data not shown). By analogy, the modeling provides distance constraints that can be applied to the $[2 \mathrm{Fe}-2 \mathrm{~S}]^{+}$and $[4 \mathrm{Fe}-4 \mathrm{~S}]^{+}$clusters in $\mathrm{HydF}^{\Delta \mathrm{EG}}$; if, for example, these two $\mathrm{FeS}$ clusters are coordinated within the dimer structure, then their interspin distance must be $\geq 25 \AA$.

HydF Quaternary Structure and Iron-Sulfur Cluster Content. The HydF ${ }^{\Delta \mathrm{EG}}$ EPR samples discussed in the previous section were subsequently analyzed via size-exclusion chromatography to examine the HydF quaternary state. The three $\mathrm{HydF}^{\Delta \mathrm{EG}}$ samples included freshly purified protein that had been treated with DTT and both as-isolated and chemically reconstituted protein that was photoreduced in the presence of DTT. Gel filtration analysis of $\mathrm{HydF}^{\mathrm{EEG}}$ reduced with DTT (Figure 6A) demonstrated that the protein sample utilized for EPR analysis (Figure 3A) existed primarily in the dimeric state, with only very low levels of the tetramer, in congruence with gel filtration studies (data not shown) that indicate DTT decreases tetramer content and increases dimer content in $\mathrm{HydF}^{\Delta \mathrm{EG}}$. Likewise, gel filtration analysis of as-isolated and chemically reconstituted $\mathrm{HydF}^{\Delta \mathrm{EG}}$ in photoreduced states (Figure 6B,C) demonstrated that these EPR samples (Figure 3B,C) both existed primarily in the dimeric state, with low levels of the tetramer. Analysis of the chemically reconstituted protein gel filtration chromatogram (Figure 6C) through simulation using Gaussian curve fitting in OriginPro Fit Peaks (pro) software provides evidence of oligomeric speciation (data not shown). Notably, a higher-molecular weight oligomeric species elutes prior to the tetramer, and a lower-molecular weight species (presumably monomeric in nature) elutes after the dimer. While we do not fully understand why these additional oligomeric states persist in chemically reconstituted $\mathrm{HydF}^{\Delta \mathrm{EG}}$, they clearly compose a minor portion of the protein analyzed by EPR, with the dominant species distinctly being the dimer state.

HydF Dimer versus Tetramer Activation of $\mathrm{HydA}^{\Delta \mathrm{EFG}}$. Given the observation that $\mathrm{HydF}^{\Delta \mathrm{EG}}$ is purified as a mixture of tetramer and dimer species that both coordinate $\mathrm{FeS}$ clusters, ${ }^{38,47}$ it was important for us to determine if expression of HydF with HydE and HydG similarly resulted in an oligomeric mixture in the as-purified protein. Gel filtration analysis of as-purified $\mathrm{HydF}^{\mathrm{EG}}$ indeed reveals that the protein exists in a mixture of dimer and tetramer states that both coordinate $\mathrm{FeS}$ cluster species (Figure S6). It was previously established that $\mathrm{HydA}^{\Delta \mathrm{EFG}}$ contains only the $[4 \mathrm{Fe}-4 \mathrm{~S}]$ cluster of the $\mathrm{H}$-cluster and is activated by delivery of the $2 \mathrm{Fe}$ 

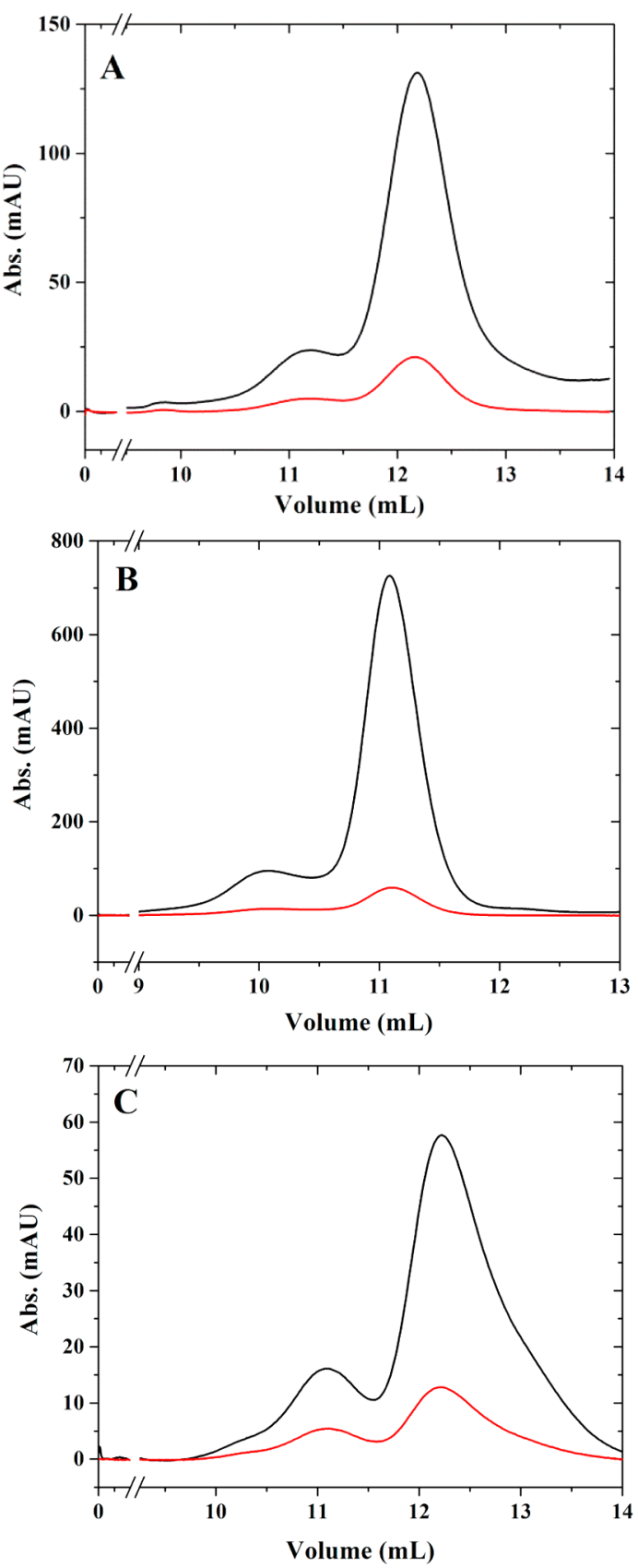

Figure 6. Gel filtration results for the $\mathrm{HydF}^{\Delta \mathrm{EG}}$ EPR samples used to measure the $T_{2}$ and $T_{1}$ values reported in Figures 4 and 5 . For each set of gel filtration spectra, the black line depicts the absorbance at $280 \mathrm{~nm}$ while the red line depicts the absorbance at $426 \mathrm{~nm}$. (A) Freshly purified $\mathrm{HydF}^{\Delta \mathrm{EG}}(104 \mu \mathrm{M}$ protein at $2.2 \pm 0.1 \mathrm{Fe}$ atoms/dimer $)$ in the presence of $5 \mathrm{mM}$ DTT. In this experiment, the tetramer fraction has a peak elution volume of $\approx 11.2 \mathrm{~mL}$ while the dimer has a peak elution volume of $\approx 12.2 \mathrm{~mL}$. (B) As-isolated $\mathrm{HydF}^{\Delta \mathrm{EG}}(600 \mu \mathrm{M}$ protein at $1.1 \pm 0.1 \mathrm{Fe}$ atoms/dimer) following photoreduction. In this experiment, the tetramer fraction has a peak elution volume of $\approx 10.1 \mathrm{~mL}$ while the dimer fraction has a peak elution volume of $\approx 11.1$ $\mathrm{mL}$. (C) Chemically reconstituted $\mathrm{HydF}^{\Delta \mathrm{EG}}(78 \mu \mathrm{M}$ protein at $5.0 \pm$ $0.7 \mathrm{Fe}$ atoms/dimer) following photoreduction. In this experiment, the tetramer fraction has a peak elution volume of $\approx 11.1 \mathrm{~mL}$ while the dimer fraction has a peak elution volume of $\approx 12.2 \mathrm{~mL}$.

subcluster from purified $\mathrm{HydF}^{\mathrm{EG}}{ }^{17,40,43,44,54,55}$ the existence of both dimer and tetramer species in as-purified $\mathrm{HydF}^{\mathrm{EG}}$ poses the question of whether these two quaternary states have distinct roles in $\mathrm{HydA}^{\triangle \mathrm{EFG}}$ activation. To investigate this issue, we tested the ability of $\mathrm{HydF}^{\mathrm{EG}}$ dimer and $\mathrm{HydF}^{\mathrm{EG}}$ tetramer fractions collected off the size-exclusion column to activate $\mathrm{HydA}^{\Delta \mathrm{EFG}}$ in vitro.

For our experiments, dimer or tetramer fractions of $\mathrm{HydF}^{\mathrm{EG}}$ were incubated with $\mathrm{HydA}^{\Delta \mathrm{EFG}}$, and hydrogen production was monitored to determine which species was better able to activate $\mathrm{HydA}^{\triangle \mathrm{EFG}}$. To isolate these oligomeric states, purified $\mathrm{HydF}^{\mathrm{EG}}$ was run through a size-exclusion column, and the peak fractions of the dimer and tetramer states were independently collected (Figure 7A). These "purified" oligomeric forms were individually incubated with $\mathrm{HydA}^{\Delta \mathrm{EFG}}$ and methyl viologen under reducing conditions and then anaerobically assayed for $\mathrm{H}_{2}$ production via gas chromatography. To provide a baseline for this experiment, control assays were also performed by monitoring $\mathrm{HydA}^{\triangle \mathrm{EFG}}$ alone and $\mathrm{HydA}^{\mathrm{BEFG}}$ incubated with $\mathrm{HydF}^{\Delta \mathrm{EG}}$; neither of these assay mixtures exhibited any measurable ability to generate $\mathrm{H}_{2}$ over the same time duration. Importantly, only the assays containing both $\mathrm{HydF}^{\mathrm{EG}}$ and $\mathrm{HydA}^{\triangle \mathrm{EFG}}$ (tested several times with variable concentrations) exhibited detectable $\mathrm{H}_{2}$ production. The assay mixtures that included $\mathrm{HydA}^{\triangle \mathrm{EFG}}$ and the $\mathrm{HydF}^{\mathrm{EG}}$ dimer showed more rapid $\mathrm{H}_{2}$ production (by an average factor of at least 3) relative to those assays that included the $\mathrm{HydF}^{\mathrm{EG}}$ tetramer (Figure $7 \mathrm{~B}$ ). To quantitatively determine the percent of dimer in the tetramer fraction, the gel filtration chromatogram was simulated as the sum of three Gaussian curves, using OriginPro Fit Peaks (pro) software. From the Gaussian for the $\mathrm{HydF}^{\mathrm{EG}}$ tetramer, the volume range collected for the tetramer fraction (9.2-9.8 $\mathrm{mL}$ ) was selected, and the area under this portion of the curve was integrated. The Gaussian for the $\mathrm{HydF}^{\mathrm{EG}}$ dimer was similarly integrated over the $9.2-9.8 \mathrm{~mL}$ volume range. The ratio of these two areas showed that approximately $6 \% \mathrm{HydF}^{\mathrm{EG}}$ dimer was present in the $\mathrm{HydF}^{\mathrm{EG}}$ tetramer fraction that was used in the activity assay (Figure 7); we take this as a lower limit for the amount of dimer in the $\mathrm{HydF}^{\mathrm{EG}}$ tetramer fraction due to the observation that these forms re-equilibrate over time. ${ }^{47}$ The low level of $\mathrm{H}_{2}$ production in the $\mathrm{HydF}^{\mathrm{EG}}$ tetramer $\mathrm{HydA}{ }^{\mathrm{EFG}}$ activation experiment can therefore be attributed to either a small amount of (active) dimer in the tetramer fraction or the low inherent activation activity of the tetramer state of HydF. Regardless, our results clearly indicate that the $\mathrm{HydF}^{\mathrm{EG}}$ dimer state of the protein readily and productively interacts with and transfers the $2 \mathrm{Fe}$ subcluster to $\mathrm{HydA}^{\Delta \mathrm{EFG}}$.

\section{DISCUSSION}

Maturation of the $\mathrm{H}$-cluster proceeds through a multistep biosynthetic process involving HydF, which plays a pivotal role as either a scaffold or a carrier protein. Our previous work has defined the nature of the FeS cluster species bound to HydF prior to its interaction with $\mathrm{HydE}$ and $\mathrm{HydG}^{40,47}$ and that $\mathrm{HydF}^{\Delta \mathrm{EG}}$ is purified as a mixture of dimeric and tetrameric states. ${ }^{47}$ While this prior work demonstrated that $\mathrm{HydF}^{\Delta \mathrm{EG}}$ coordinated redox-active $[4 \mathrm{Fe}-4 \mathrm{~S}]^{2+/+}$ and $[2 \mathrm{Fe}-2 \mathrm{~S}]^{2+/+}$ clusters, ${ }^{47}$ it did not resolve $[4 \mathrm{Fe}-4 \mathrm{~S}]^{+}$and $[2 \mathrm{Fe}-2 \mathrm{~S}]^{+}$cluster proximity, nor did it examine the $\mathrm{FeS}$ cluster state(s) associated with the two respective quaternary forms of $\mathrm{HydF}^{\Delta \mathrm{EG}}$. Moreover, prior work did not examine the roles of dimeric or

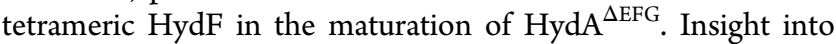
these questions is presented herein, allowing us to refine the steps involving HydF both before and after interaction with HydE and HydG. 

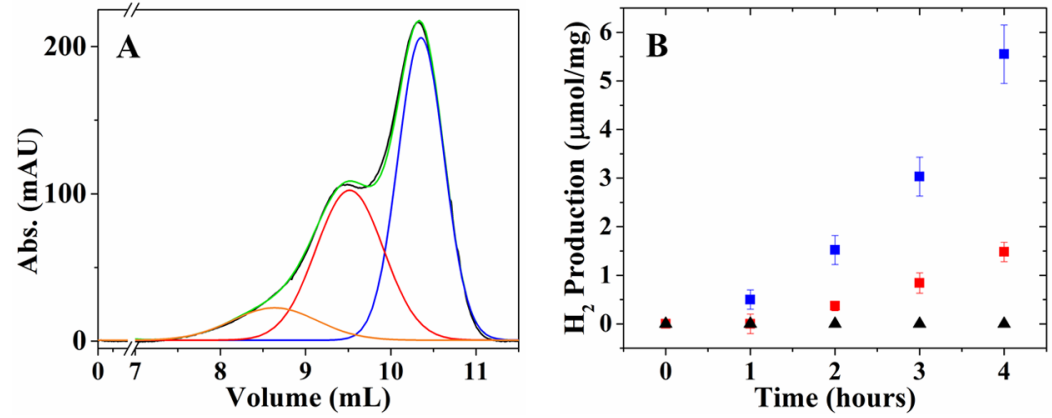

Figure 7. $\mathrm{HydA}^{\triangle \mathrm{EFG}}$ activation upon exposure to $\mathrm{HydF}^{\mathrm{EG}}$ dimer or $\mathrm{HydF}^{\mathrm{EG}}$ tetramer gel filtration fractions. (A) Gel filtration of as-purified $\mathrm{HydF} \mathrm{F}^{\mathrm{EG}}$ that guided the dimer and tetramer fraction collection for the activity assay. The black trace represents the experimental absorbance at $280 \mathrm{~nm}$; simulations of the composite spectrum (green), the dimer fraction (blue), the tetramer fraction (red), and an additional component (orange) are additionally displayed. In this experiment, the volumes collected for the tetramer and dimer fractions were 9.2-9.8 and 10.1-10.7 mL, respectively;

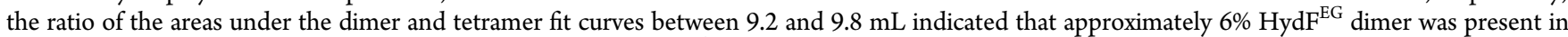
the fraction that was designated as $\mathrm{HydF}^{\mathrm{EG}}$ tetramer. (B) $\mathrm{HydA}{ }^{\Delta \mathrm{EFG}}$ activation resulting from incubation with the $\mathrm{HydF}^{\mathrm{EG}}$ tetramer or dimer, as described for panel A (see also Figure S6). The symbols refer to $\mathrm{HydA}^{\Delta \mathrm{EFG}}$ incubated with $\mathrm{HydF}^{\Delta \mathrm{EG}}$ (black triangles), HydA ${ }^{\Delta \mathrm{EFG}}$ incubated with $\mathrm{HydF}^{\mathrm{EG}}$ dimer (blue squares), and $\mathrm{HydA}^{\triangle \mathrm{EFG}}$ incubated with $\mathrm{HydF}^{\mathrm{EG}}$ tetramer (red squares). In these activity assays, the concentration of $\mathrm{HydA}^{\mathrm{BEFG}}$ was $0.5 \mu \mathrm{M}$ while the concentrations were $6.2 \mu \mathrm{M}$ for the $\mathrm{HydF}^{\mathrm{EG}}$ dimer (or $12.4 \mu \mathrm{M}$ active sites) and $6.2 \mu \mathrm{M}$ for the $\mathrm{HydF}^{\mathrm{EG}}$ tetramer (or $24.8 \mu \mathrm{M}$ active sites).

The proximity of the individual subunit $\mathrm{CXHX}_{46-53} \mathrm{CXXC}$ motifs in the tetramer structure of HydF (a dimer of dimers) is such that these putative ligands approach one another, as evidenced by interdisulfide bond formation between pairs of Cys302 residues from different dimers. ${ }^{38}$ This leads to the possibility that $\mathrm{FeS}$ cluster coordination in $\mathrm{HydF}^{\Delta \mathrm{EG}}$ involves ligand sets from two different monomers. Examination of the intersubunit distances between pairs of corresponding residues within the $\mathrm{CXHX}_{46-53} \mathrm{CXXC}$ motifs in the dimer structure of $\mathrm{HydF}^{\mathrm{AEG}}$ (Figure 2) shows that these residues are $30-41 \AA$ apart (Figure S7). These ligands are located on loop regions (Figure 2) that could allow for considerable flexibility in terms of FeS cluster coordination, rearrangement, and transfer. ${ }^{31,38}$ Moreover, the domain architecture of the dimer state reveals the possibility for substantial structural flexibility given the existence of S-shaped loop regions of amino acids that form links between domains I and II and between domains II and III. $^{38,76}$ The resulting conformational flexibility in the dimer could allow formation of a collapsed structure, decreasing the distance between corresponding residues within the $\mathrm{CXH}$ $\mathrm{X}_{46-53} \mathrm{CXXC}$ motifs to 21-23 $\AA$ (Figure S7). These observations suggest that $\mathrm{FeS}$ cluster coordination in dimeric HydF could be accomplished via interfacial binding, thus providing a mechanism for establishing interaction between the two monomeric subunits beyond the dimerization domain. ${ }^{31,76}$

Freshly purified $\mathrm{HydF}^{\Delta \mathrm{EG}}$ predominantly exists as a dimer in solution, ${ }^{38,47}$ and sample handling has been demonstrated to perturb the dimer:tetramer ratio. ${ }^{47}$ To examine the FeS clusterloaded forms of $\mathrm{HydF}^{\mathrm{EEG}}$ via low-temperature EPR, we treated the protein with different chemical reducing agents, thus generating $\mathrm{HydF}^{\Delta \mathrm{EG}}$ that either exhibited only a $[2 \mathrm{Fe}-2 \mathrm{~S}]^{+}$ cluster signal (Figure $3 \mathrm{~A}$ ) or showed overlapping $[2 \mathrm{Fe}-2 \mathrm{~S}]^{+}$ and $[4 \mathrm{Fe}-4 \mathrm{~S}]^{+}$cluster signals (Figure $\left.3 \mathrm{~B}, \mathrm{C}\right) .^{47}$ An important observation drawn in this study is that the spectroscopic samples of $\mathrm{HydF}^{\Delta \mathrm{EG}}$ that exhibited either only $[2 \mathrm{Fe}-2 \mathrm{~S}]^{+}$or both $[4 \mathrm{Fe}-4 \mathrm{~S}]^{+}$and $[2 \mathrm{Fe}-2 \mathrm{~S}]^{+}$cluster signals are predominantly dimeric in nature (Figure 6). While the current data cannot rule out the possibility that the tetramer coordinates either one or both of these FeS clusters, it is clear from the abundance of the dimer species in the EPR samples that the measured electron spin relaxation properties for the $[2 \mathrm{Fe}-2 \mathrm{~S}]^{+}$and $[4 \mathrm{Fe}-4 \mathrm{~S}]^{+}$ cluster signals associated with $\mathrm{HydF}^{\Delta \mathrm{EG}}$ (Figures 4 and 5 and Table 1) correspond primarily with the dimeric state of the protein.

The relaxation enhancement measurements reported herein demonstrate that the $[4 \mathrm{Fe}-4 \mathrm{~S}]^{+}$cluster does not alter the relaxation properties of the $[2 \mathrm{Fe}-2 \mathrm{~S}]^{+}$cluster in photoreduced $\mathrm{HydF}^{\Delta \mathrm{EG}}$. A comparison of the temperature-dependent inversion recovery $T_{1}$ relaxation rates in Figure 5 shows similar values for $[2 \mathrm{Fe}-2 \mathrm{~S}]^{+}$cluster signals in $\mathrm{HydF}^{\mathrm{EEG}}$ samples in either DTT alone or DTT and photoreduction conditions. Photoreduced as-isolated $\mathrm{HydF}^{\mathrm{EEG}}$ would be expected to exhibit faster relaxation rates if relaxation enhancement of $[2 \mathrm{Fe}-$ $2 \mathrm{~S}]^{+}$by proximate $[4 \mathrm{Fe}-4 \mathrm{~S}]^{+}$clusters existed. It should be stated that overexpression of $\mathrm{HydF}^{\Delta \mathrm{EG}}$ from various sources commonly yields a protein that contains a small complement of FeS clusters. ${ }^{38-40,43,47,77-80}$ This may be due in part to the inherent lability of these clusters as an intrinsic function of this protein in first scaffolding the assembly of the $2 \mathrm{Fe}$ subcluster followed by transfer of the manufactured $2 \mathrm{Fe}$ subcluster to $\mathrm{HydA}^{\Delta \mathrm{EFG}}$; this inherent FeS cluster lability makes characterization of the relevant $\mathrm{FeS}$ cluster states in this system challenging. ${ }^{47}$ Along these lines, we probed the variable of low FeS cluster occupancy as a potential source of the lack of enhanced relaxation in photoreduced as-isolated $\mathrm{HydF}^{\Delta \mathrm{EG}}$ by preparing and analyzing a chemically reconstituted sample of $\mathrm{HydF}^{\Delta \mathrm{EG}}$. Reconstitution of $\mathrm{HydF}^{\Delta \mathrm{EG}}$ resulted in a significant increase in the iron number associated with the protein to $5.0 \pm$ $0.7 \mathrm{Fe}$ atoms/dimer, and examination of the $\mathrm{CW}$ spectra for the photoreduced protein reveals that chemically reconstituted $\mathrm{HydF}^{\Delta \mathrm{EG}}$ exhibits greater $[2 \mathrm{Fe}-2 \mathrm{~S}]^{+}$cluster occupancy, relative to as-isolated $\mathrm{HydF}^{\Delta \mathrm{EG}}$ [compare the relative peak height ratios for the $g=2.006$ signal $\left([2 \mathrm{Fe}-2 \mathrm{~S}]^{+}\right)$to the $g=2.050$ signal $\left([4 \mathrm{Fe}-4 \mathrm{~S}]^{+}\right)$in panels $\mathrm{B}$ and $\mathrm{C}$ of Figure 3$]$. Importantly, inversion recovery $T_{1}$ relaxation rates for chemically reconstituted, photoreduced $\mathrm{HydF}^{\Delta \mathrm{EG}}$ showed no significant differences relative to as-isolated, photoreduced $\mathrm{HydF}^{\Delta \mathrm{EG}}$. While we acknowledge that it is possible that the $\mathrm{FeS}$ cluster coordination state of HydF under physiological conditions (during maturation of HydA) may be distinct from the overexpressed protein that we are currently analyzing, the collective results reported herein do not support any $\mathrm{FeS}$ cluster interspin 
relaxation effects in dimeric $\mathrm{HydF}^{\Delta \mathrm{EG}}$. Moreover, the ability to observe $[2 \mathrm{Fe}-2 \mathrm{~S}]^{+}$cluster relaxation enhancement in $\mathrm{HydE}$ via its proximity to a $[3 \mathrm{Fe}-4 \mathrm{~S}]^{+}$cluster (with an interspin distance $r$ of $22 \AA$ ) allows us to conclude that if the $[2 \mathrm{Fe}-2 \mathrm{~S}]^{+}$and $[4 \mathrm{Fe}-$ $4 \mathrm{~S}]^{+}$clusters are both coordinated to dimeric $\mathrm{HydF}^{\Delta \mathrm{EG}}$, that they must be at a distance of $\geq 25 \AA$ (Figure S5).

The simulations of relaxation rates for HydE help to establish that the $[2 \mathrm{Fe}-2 \mathrm{~S}]^{+}$cluster in these $\mathrm{HydF}^{\Delta \mathrm{EG}}$ samples is not near, or directly bridged to, the $[4 \mathrm{Fe}-4 \mathrm{~S}]^{+}$cluster, which in turn suggests that the $[2 \mathrm{Fe}-2 \mathrm{~S}]^{+}$and $[4 \mathrm{Fe}-4 \mathrm{~S}]^{+}$clusters are coordinated within different monomeric subunits of the dimer form or that one population of dimeric $\mathrm{HydF}^{\Delta \mathrm{EG}}$ contains only the $[2 \mathrm{Fe}-2 \mathrm{~S}]^{+}$while another population of the dimeric enzyme contains only the $[4 \mathrm{Fe}-4 \mathrm{~S}]^{+}$cluster. Importantly, the advanced EPR measurements presented herein have helped to define the electronic properties of the $[2 \mathrm{Fe}-2 \mathrm{~S}]^{+}$ cluster-coordinated state of $\mathrm{HydF}^{\Delta \mathrm{EG}}$. Interestingly, the $[2 \mathrm{Fe}$ $2 \mathrm{~S}]^{+}$cluster signal associated with dimeric $\mathrm{HydF}^{\Delta \mathrm{EG}}$ exhibits weak spin-orbit coupling that is reflected in the relatively small $g$ value anisotropy (0.046) for this signal; this observation coupled to the small values of $C_{\text {Ram }}$ and $C_{\text {orb }}$ (Table 1) that were utilized to generate the fit lines in Figure 5 suggests that the $[2 \mathrm{Fe}-2 \mathrm{~S}]^{+}$cluster in $\mathrm{HydF}^{\Delta \mathrm{EG}}$ is in a rigid structural environment. ${ }^{58}$ While the exact ligand environment of the [2Fe-2S] cluster in HydF is unknown, the observation herein that the $[2 \mathrm{Fe}-2 \mathrm{~S}]^{+}$cluster resides within a subunit of the dimer supports residues of the conserved $\mathrm{CXHX}_{46-53} \mathrm{CXXC}$ motif as coordinating this species.

It has been previously proposed that the $[2 \mathrm{Fe}-2 \mathrm{~S}]$ cluster was the scaffold cluster for $2 \mathrm{Fe}$ subcluster assembly via delivery of the DTMA, CO, and $\mathrm{CN}^{-}$ligands synthesized by HydE and HydG (Figure 1B). ${ }^{40}$ Alternatively, the [2Fe-2S] cluster either could be involved in electron transfer steps during $2 \mathrm{Fe}$ subcluster assembly or may act as a placeholder for HydGderived $\mathrm{Fe}(\mathrm{CO})_{2}(\mathrm{CN})$ Cys synthons. ${ }^{24,32,33,35}$ Given that the cluster binding sites in tetrameric HydF appear to be less accessible for direct protein-protein cluster transfer, it has been proposed that HydE and HydG interact with the dimeric form of $\mathrm{HydF}$ where the cluster binding sites are more exposed; these interactions are thought to occur stepwise and may be gated by GTP binding and hydrolysis. ${ }^{38,40-42}$ Moreover, our results show that the $\mathrm{HydF}^{\mathrm{EG}}$ dimer state exhibits $\mathrm{HydA}^{\Delta \mathrm{EFG}}$ activation capability significantly better than that of the $\mathrm{HydF}^{\mathrm{EG}}$ tetramer state (Figure 7), thereby establishing that $2 \mathrm{Fe}$ subcluster transfer to $\mathrm{HydA}^{\mathrm{EFG}}$ more readily occurs from the dimer state of $\mathrm{HydF}^{\mathrm{EG}}$ (Figure 8). Together, the results help to establish a vital role for the dimeric form of HydF (and its associated $\mathrm{FeS}$ cluster states) in the biosynthesis of the $2 \mathrm{Fe}$ subcluster.

\section{CONCLUSIONS}

As-purified HydF exists as a mixture of dimer and tetramer forms that each coordinate $\mathrm{FeS}$ cluster species. Until now, no information regarding the roles of either of these quaternary states of HydF during $\mathrm{H}$-cluster biogenesis has existed. Our results show that the dimeric $\mathrm{HydF}^{\mathrm{EG}}$ productively interacts with and transfers the $2 \mathrm{Fe}$ subcluster to $\mathrm{HydA}^{\mathrm{BFG}}$. The role of the tetramer species in H-cluster maturation is, however, still unresolved. The observation that the dimer and tetramer states exist in a dynamic equilibrium with one another ${ }^{47}$ suggests the possibility of a physiologically relevant role for the tetramer during $\mathrm{H}$-cluster maturation; it is plausible that this form may act to protect the $2 \mathrm{Fe}$ subcluster when copy numbers of HydA
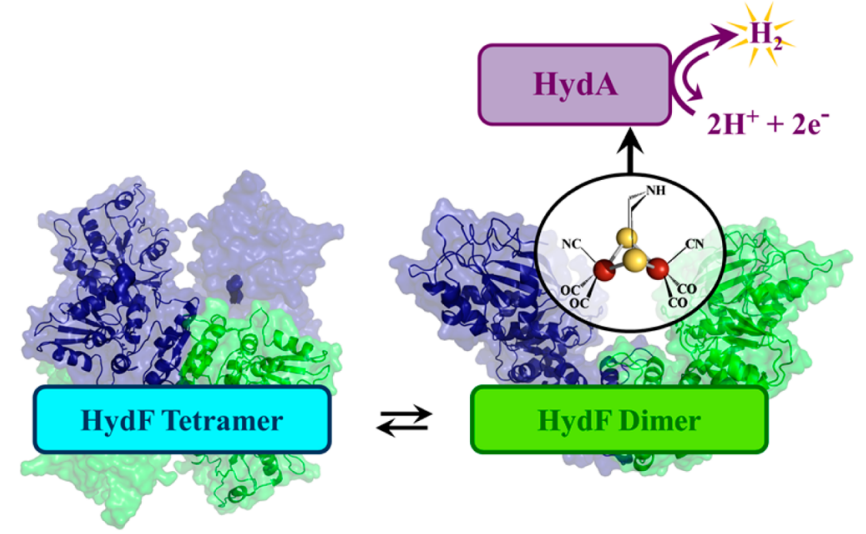

Figure 8. Hypothetical maturation scheme depicting the transfer of the $2 \mathrm{Fe}$ subcluster precursor from the $\mathrm{HydF}^{\mathrm{EG}}$ dimer to $\mathrm{HydA}^{\Delta \mathrm{EFG}}$. For the HydF tetramer, one dimeric subunit is depicted as a transparent surface map while the other dimeric subunit is illustrated by a transparent surface surrounding a nontransparent cartoon depiction of the structure. In both cases, one monomeric subunit is colored green and the other monomeric subunit is colored blue. For the $\mathrm{HydF}^{\mathrm{EG}}$ dimer appearing on the right, the $2 \mathrm{Fe}$ subcluster H-cluster precursor is depicted on this protomer and shown to be transferred to $\mathrm{HydA}^{\triangle \mathrm{EFG}}$ to allow for maturation of HydA. The arrows indicate that the dimeric and tetrameric protomers are in dynamic equilibrium. The color scheme for the FeS clusters depicted in this figure is as follows: iron, rust; sulfur, yellow.

in the cell are low. Prior to interacting with HydE and HydG to build the $2 \mathrm{Fe}$ subcluster, $\mathrm{HydF}^{\Delta \mathrm{EG}}$ is primarily dimeric and binds both $[4 \mathrm{Fe}-4 \mathrm{~S}]$ and $[2 \mathrm{Fe}-2 \mathrm{~S}]$ clusters. Our results show that these clusters are not, however, in the proximity of each other and thus are not necessarily poised to generate an $\mathrm{H}$ cluster-like $6 \mathrm{Fe}$ species simply upon ligand delivery by HydE and HydG. The implications of these results in the overall scheme of $\mathrm{H}$-cluster maturation await further studies.

\section{ASSOCIATED CONTENT}

\section{S Supporting Information}

The Supporting Information is available free of charge on the ACS Publications website at DOI: 10.1021/acs.biochem.7b00169.

Low-temperature X-band CW EPR spectra for as-purified PFL-AE and as-reconstituted $\mathrm{HydE}$; temperature dependence of X-band $\mathrm{CW}$ spectra of $[2 \mathrm{Fe}-2 \mathrm{~S}]^{+}$cluster signals in as-purified PFL-AE and freshly purified $\mathrm{HydF}^{\mathrm{EG}}$ in the presence of $5 \mathrm{mM}$ dithiothreitol; Xband field-swept echo-detected spectra obtained with 40 ns $\pi / 2$ pulses for as-purified PFL-AE, as-reconstituted $\mathrm{HydE}$, as-isolated $\mathrm{HydF}^{\Delta \mathrm{EG}}$ following photoreduction, and freshly purified $\mathrm{HydF}^{\Delta \mathrm{EG}}$ in the presence of $5 \mathrm{mM}$ dithiothreitol; inversion recovery data at $g_{\perp}$ for the $[2 \mathrm{Fe}$ $2 \mathrm{~S}]^{+}$cluster in as-reconstituted $\mathrm{HydE}$ at $40 \mathrm{~K}$ and calculations with MENOSR; illustration of the $\mathrm{FeS}$ cluster content of $\mathrm{HydF}^{\mathrm{EG}}$ tetramer and $\mathrm{HydF}^{\mathrm{EG}}$ dimer fractions utilized in $\mathrm{HydA}^{\Delta \mathrm{EFG}}$ activation assays; and a collapsed model of the $\mathrm{HydF}^{\Delta \mathrm{EG}}$ dimer structure (PDF)

\section{AUTHOR INFORMATION}

\section{Corresponding Author}

*E-mail: jbroderick@chemistry.montana.edu. Phone: (406) 994-6160. 


\section{ORCID}

Joan B. Broderick: 0000-0001-7057-9124

\section{Author Contributions}

E.M.S. and A.S.B. contributed equally to this work.

\section{Funding}

This work was supported by U.S. Department of Energy Grant DE-FG02-10ER16194 to J.B.B. and E.M.S. (all work on the Hyd proteins) and the National Institutes of Health Grant R01 GM54608 to J.B.B. (work on PFL-AE). Work at the University of Denver EPR Center was supported by the University of Denver. J.N.B. thanks the U.S. Air Force Academy Faculty Pipeline Ph.D. Program for its sponsorship.

\section{Notes}

The authors declare no competing financial interest.

\section{ABBREVIATIONS}

C.a., Clostridium acetobutylicum; C.p., Clostridium pasteurianum; $\mathrm{C} \mathrm{I}$, [FeFe]-hydrogenase I from C. pasteurianum; EPR, electron paramagnetic resonance spectroscopy; FeS, iron sulfur; FTIR, Fourier transform infrared spectroscopy; GTP, guanosine triphosphate; HydA, [FeFe]-hydrogenase; HydA ${ }^{\Delta \mathrm{EFG}}$, HydA expressed without HydE, HydF, and HydG maturases; HydA $^{\mathrm{EFG}}$, HydA co-expressed with HydE, HydF, and HydG maturases; $\mathrm{HydF}^{\Delta \mathrm{EG}}$, maturase protein $\mathrm{HydF}$ expressed without $\mathrm{HydE}$ or $\mathrm{HydG}$; $\mathrm{HydF}^{\mathrm{EG}}$, maturase protein $\mathrm{HydF}$ co-expressed with HydE and HydG; ISC, iron-sulfur cluster assembly machinery; LMCT, ligand to metal charge transfer; PDB, Protein Data Bank; PFL-AE, pyruvate formate lyase-activating enzyme; PMSF, phenylmethanesulfonyl fluoride; Ras, rat sarcoma; SAM, S-adenosyl-L-methionine; S.o., Shewanella oneidensis; T.m., Thermotoga maritima; T.n., Thermotoga neopolitana; WT, wild type; XAS, X-ray absorption spectroscopy.

\section{REFERENCES}

(1) Peters, J. W., Schut, G. J., Boyd, E. S., Mulder, D. W., Shepard, E. M., Broderick, J. B., King, P. W., and Adams, M. W. (2015) [FeFe]and $[\mathrm{NiFe}]$-hydrogenase diversity, mechanism, and maturation. Biochim. Biophys. Acta, Mol. Cell Res. 1853, 1350-1369.

(2) Adams, M. W. (1990) The structure and mechanism of ironhydrogenases. Biochim. Biophys. Acta, Bioenerg. 1020, 115-145.

(3) Fritsch, J., Scheerer, P., Frielingsdorf, S., Kroschinsky, S., Friedrich, B., Lenz, O., and Spahn, C. M. (2011) The crystal structure of an oxygen-tolerant hydrogenase uncovers a novel iron-sulphur centre. Nature 479, 249-252.

(4) Goris, T., Wait, A. F., Saggu, M., Fritsch, J., Heidary, N., Stein, M., Zebger, I., Lendzian, F., Armstrong, F. A., Friedrich, B., and Lenz, O. (2011) A unique iron-sulfur cluster is crucial for oxygen tolerance of a [NiFe]-hydrogenase. Nat. Chem. Biol. 7, 310-318.

(5) Madden, C., Vaughn, M. D., Diez-Perez, I., Brown, K. A., King, P. W., Gust, D., Moore, A. L., and Moore, T. A. (2012) Catalytic turnover of [FeFe]-hydrogenase based on single-molecule imaging. J. Am. Chem. Soc. 134, 1577-1582.

(6) Swanson, K. D., Ratzloff, M. W., Mulder, D. W., Artz, J. H., Ghose, S., Hoffman, A., White, S., Zadvornyy, O. A., Broderick, J. B., Bothner, B., King, P. W., and Peters, J. W. (2015) [FeFe]-hydrogenase oxygen inactivation is initiated at the $\mathrm{H}$-cluster $2 \mathrm{Fe}$ subcluster. J. Am. Chem. Soc. 137, 1809-1816.

(7) Lubitz, W., Ogata, H., Rudiger, O., and Reijerse, E. (2014) Hydrogenases. Chem. Rev. 114, 4081-4148.

(8) Peters, J. W., Lanzilotta, W. N., Lemon, B. J., and Seefeldt, L. C. (1998) X-ray crystal structure of the Fe-only hydrogenase (CpI) from Clostridium pasteurianum to 1.8 angstrom resolution. Science 282, $1853-1858$.

(9) Nicolet, Y., Piras, C., Legrand, P., Hatchikian, C. E., and Fontecilla-Camps, J. C. (1999) Desulfovibrio desulfuricans iron hydrogenase: the structure shows unusual coordination to an active site Fe binuclear center. Structure 7, 13-23.

(10) Chen, Z., Lemon, B. J., Huang, S., Swartz, D. J., Peters, J. W., and Bagley, K. A. (2002) Infrared studies of the CO-inhibited form of the Fe-only hydrogenase from Clostridium pasteurianum I: examination of its light sensitivity at cryogenic temperatures. Biochemistry 41, 2036-2043.

(11) Berggren, G., Adamska, A., Lambertz, C., Simmons, T. R, Esselborn, J., Atta, M., Gambarelli, S., Mouesca, J. M., Reijerse, E., Lubitz, W., Happe, T., Artero, V., and Fontecave, M. (2013) Biomimetic assembly and activation of $[\mathrm{FeFe}]$-hydrogenases. Nature 499, 66-69.

(12) Posewitz, M. C., King, P. W., Smolinski, S. L., Zhang, L., Seibert, M., and Ghirardi, M. L. (2004) Discovery of two novel radical $S$ adenosylmethionine proteins required for the assembly of an active [Fe] hydrogenase. J. Biol. Chem. 279, 25711-25720.

(13) King, P. W., Posewitz, M. C., Ghirardi, M. L., and Seibert, M. (2006) Functional studies of $[\mathrm{FeFe}]$ hydrogenase maturation in an Escherichia coli biosynthetic system. J. Bacteriol. 188, 2163-2172.

(14) McGlynn, S. E., Ruebush, S. S., Naumov, A., Nagy, L. E., Dubini, A., King, P. W., Broderick, J. B., Posewitz, M. C., and Peters, J. W. (2007) In vitro activation of [FeFe] hydrogenase: new insights into hydrogenase maturation. J. Biol. Inorg. Chem. 12, 443-447.

(15) Rubach, J. K., Brazzolotto, X., Gaillard, J., and Fontecave, M. (2005) Biochemical characterization of the HydE and HydG iron-only hydrogenase maturation enzymes from Thermatoga maritima. FEBS Lett. 579, 5055-5060.

(16) Shepard, E. M., Boyd, E. S., Broderick, J. B., and Peters, J. W. (2011) Biosynthesis of complex iron-sulfur enzymes. Curr. Opin. Chem. Biol. 15, 319-327.

(17) Peters, J. W., and Broderick, J. B. (2012) Emerging paradigms for complex iron-sulfur cofactor assembly and insertion. Annu. Rev. Biochem. 81, 429-450.

(18) Byer, A. S., Shepard, E. M., Peters, J. W., and Broderick, J. B. (2015) Radical S-adenosyl-L-methionine chemistry in the synthesis of hydrogenase and nitrogenase metal cofactors. J. Biol. Chem. 290, 3987-3994.

(19) Broderick, J. B., Duffus, B. R., Duschene, K. S., and Shepard, E. M. (2014) Radical S-Adenosylmethionine Enzymes. Chem. Rev. 114, $4229-4317$.

(20) Betz, J. N., Boswell, N. W., Fugate, C. J., Holliday, G. L., Akiva, E., Scott, A. G., Babbitt, P. C., Peters, J. W., Shepard, E. M., and Broderick, J. B. (2015) [FeFe]-hydrogenase maturation: insights into the role HydE plays in dithiomethylamine biosynthesis. Biochemistry $54,1807-1818$

(21) Li, H., and Rauchfuss, T. B. (2002) Iron carbonyl sulfides, formaldehyde, and amines condense to give the proposed azadithiolate cofactor of the Fe-only hydrogenases. J. Am. Chem. Soc. 124, 726-727.

(22) Rohac, R., Amara, P., Benjdia, A., Martin, L., Ruffie, P., Favier, A., Berteau, O., Mouesca, J. M., Fontecilla-Camps, J. C., and Nicolet, Y. (2016) Carbon-sulfur bond-forming reaction catalysed by the radical SAM enzyme HydE. Nat. Chem. 8, 491-500.

(23) Nicolet, Y., Pagnier, A., Zeppieri, L., Martin, L., Amara, P., and Fontecilla-Camps, J. C. (2015) Crystal structure of HydG from Carboxydothermus hydrogenoformans: a trifunctional [FeFe]-hydrogenase maturase. ChemBioChem 16, 397-402.

(24) Dinis, P., Suess, D. L., Fox, S. J., Harmer, J. E., Driesener, R. C., De La Paz, L., Swartz, J. R., Essex, J. W., Britt, R. D., and Roach, P. L. (2015) X-ray crystallographic and EPR spectroscopic analysis of HydG, a maturase in [FeFe]-hydrogenase H-cluster assembly. Proc. Natl. Acad. Sci. U. S. A. 112, 1362-1367.

(25) Pilet, E., Nicolet, Y., Mathevon, C., Douki, T., Fontecilla-Camps, J. C., and Fontecave, M. (2009) The role of the maturase HydG in $[\mathrm{FeFe}]$-hydrogenase active site synthesis and assembly. FEBS Lett. 583, 506-511.

(26) Driesener, R. C., Challand, M. R., McGlynn, S. E., Shepard, E. M., Boyd, E. S., Broderick, J. B., Peters, J. W., and Roach, P. L. (2010) $[\mathrm{FeFe}]$-hydrogenase cyanide ligands derived from $S$-adenosylmethio- 
nine-dependent cleavage of tyrosine. Angew. Chem., Int. Ed. 49, 16871690.

(27) Duffus, B. R., Ghose, S., Peters, J. W., and Broderick, J. B. (2014) Reversible $\mathrm{H}$ atom abstraction catalyzed by the radical $S$ adenosylmethionine enzyme HydG. J. Am. Chem. Soc. 136, 1308613089.

(28) Kuchenreuther, J. M., Myers, W. K., Stich, T. A., George, S. J., Nejatyjahromy, Y., Swartz, J. R., and Britt, R. D. (2013) A radical intermediate in tyrosine scission to the $\mathrm{CO}$ and $\mathrm{CN}^{-}$ligands of [FeFe]-hydrogenase. Science 342, 472-475.

(29) Shepard, E. M., Duffus, B. R., George, S. J., McGlynn, S. E., Challand, M. R., Swanson, K. D., Roach, P. L., Cramer, S. P., Peters, J. W., and Broderick, J. B. (2010) [FeFe]-hydrogenase maturation: HydG-catalyzed synthesis of carbon monoxide. J. Am. Chem. Soc. 132, 9247-9249.

(30) Driesener, R. C., Duffus, B. R., Shepard, E. M., Bruzas, I. R, Duschene, K. S., Coleman, N. J., Marrison, A. P., Salvadori, E., Kay, C. W., Peters, J. W., Broderick, J. B., and Roach, P. L. (2013) Biochemical and Kinetic Characterization of Radical S-Adenosyl-1-methionine Enzyme HydG. Biochemistry 52, 8696-8707.

(31) Shepard, E. M., Mus, F., Betz, J. N., Byer, A. S., Duffus, B. R., Peters, J. W., and Broderick, J. B. (2014) [FeFe]-Hydrogenase Maturation. Biochemistry 53, 4090-4104.

(32) Kuchenreuther, J. M., Myers, W. K., Suess, D. L., Stich, T. A., Pelmenschikov, V., Shiigi, S. A., Cramer, S. P., Swartz, J. R., Britt, R. D., and George, S. J. (2014) The HydG enzyme generates an $\mathrm{Fe}(\mathrm{CO})_{2}(\mathrm{CN})$ synthon in assembly of the $[\mathrm{FeFe}]$-hydrogenase $\mathrm{H}$ cluster. Science 343, 424-427.

(33) Suess, D. L., Burstel, I., De La Paz, L., Kuchenreuther, J. M., Pham, C. C., Cramer, S. P., Swartz, J. R., and Britt, R. D. (2015) Cysteine as a ligand platform in the biosynthesis of the $\mathrm{FeFe}$ hydrogenase H cluster. Proc. Natl. Acad. Sci. U. S. A. 112, 1145511460.

(34) Pagnier, A., Martin, L., Zeppieri, L., Nicolet, Y., and FontecillaCamps, J. C. (2016) CO and $\mathrm{CN}^{-}$syntheses by [FeFe]-hydrogenase maturase HydG are catalytically differentiated events. Proc. Natl. Acad. Sci. U. S. A. 113, 104-109.

(35) Suess, D. L., Pham, C. C., Burstel, I., Swartz, J. R., Cramer, S. P., and Britt, R. D. (2016) The Radical SAM Enzyme HydG Requires Cysteine and a Dangler Iron for Generating an Organometallic Precursor to the [FeFe]-Hydrogenase H-Cluster. J. Am. Chem. Soc. 138, 1146-1149.

(36) Dinis, P., Wieckowski, B. M., and Roach, P. L. (2016) Metallocofactor assembly for [FeFe]-hydrogenases. Curr. Opin. Struct. Biol. 41, 90-97.

(37) Cox, A. D., and Der, C. J. (2010) Ras history: The saga continues. Small GTPases 1, 2-27.

(38) Cendron, L., Berto, P., D’Adamo, S., Vallese, F., Govoni, C., Posewitz, M. C., Giacometti, G. M., Costantini, P., and Zanotti, G. (2011) Crystal structure of HydF scaffold protein provides insights into $[\mathrm{FeFe}]$-hydrogenase maturation. J. Biol. Chem. 286, 4394443950.

(39) Brazzolotto, X., Rubach, J. K., Gaillard, J., Gambarelli, S., Atta, M., and Fontecave, M. (2006) The [Fe-Fe]-hydrogenase maturation protein HydF from Thermotoga maritima is a GTPase with an ironsulfur cluster. J. Biol. Chem. 281, 769-774.

(40) Shepard, E. M., McGlynn, S. E., Bueling, A. L., Grady-Smith, C. S., George, S. J., Winslow, M. A., Cramer, S. P., Peters, J. W., and Broderick, J. B. (2010) Synthesis of the $2 \mathrm{Fe}$ subcluster of the [FeFe]hydrogenase $\mathrm{H}$ cluster on the HydF scaffold. Proc. Natl. Acad. Sci. U. S. A. 107, 10448-10453.

(41) Vallese, F., Berto, P., Ruzzene, M., Cendron, L., Sarno, S., De Rosa, E., Giacometti, G. M., and Costantini, P. (2012) Biochemical analysis of the interactions between the proteins involved in the [FeFe]-hydrogenase maturation process. J. Biol. Chem. 287, 3654436555 .

(42) Galazzo, L., Maso, L., De Rosa, E., Bortolus, M., Doni, D., Acquasaliente, L., Filippis, V., Costantini, P., and Carbonera, D. (2017) Identifying conformational changes with site-directed spin labeling reveals that the GTPase domain of HydF is a molecular switch. Sci. Rep. 7, 1714.

(43) McGlynn, S. E., Shepard, E. M., Winslow, M. A., Naumov, A. V., Duschene, K. S., Posewitz, M. C., Broderick, W. E., Broderick, J. B., and Peters, J. W. (2008) HydF as a scaffold protein in [FeFe]hydrogenase H-cluster biosynthesis. FEBS Lett. 582, 2183-2187.

(44) Mulder, D. W., Shepard, E. M., Meuser, J. E., Joshi, N., King, P. W., Posewitz, M. C., Broderick, J. B., and Peters, J. W. (2011) Insights into $[\mathrm{FeFe}]$-hydrogenase structure, mechanism, and maturation. Structure 19, 1038-1052.

(45) Czech, I., Silakov, A., Lubitz, W., and Happe, T. (2010) The $[\mathrm{FeFe}]$-hydrogenase maturase $\mathrm{HydF}$ from Clostridium acetobutylicum contains a $\mathrm{CO}$ and $\mathrm{CN}^{-}$ligated iron cofactor. FEBS Lett. 584, 638642.

(46) Esselborn, J., Lambertz, C., Adamska-Venkatesh, A., Simmons, T., Berggren, G., Noth, J., Siebel, J., Hemschemeier, A., Artero, V., Reijerse, E., Fontecave, M., Lubitz, W., and Happe, T. (2013) Spontaneous activation of $[\mathrm{FeFe}]$-hydrogenases by an inorganic $[2 \mathrm{Fe}]$ active site mimic. Nat. Chem. Biol. 9, 607-609.

(47) Shepard, E. M., Byer, A. S., Betz, J. N., Peters, J. W., and Broderick, J. B. (2016) A Redox Active [2Fe-2S] Cluster on the Hydrogenase Maturase HydF. Biochemistry 55, 3514-3527.

(48) Broderick, J. B., Henshaw, T. F., Cheek, J., Wojtuszewski, K., Smith, S. R., Trojan, M. R., McGhan, R. M., Kopf, A., Kibbey, M., and Broderick, W. E. (2000) Pyruvate formate-lyase-activating enzyme: strictly anaerobic isolation yields active enzyme containing a $[3 \mathrm{Fe}-4 \mathrm{~S}]^{+}$ cluster. Biochem. Biophys. Res. Commun. 269, 451-456.

(49) Henshaw, T. F., Cheek, J., and Broderick, J. B. (2000) The [4Fe$4 \mathrm{~S}] 1+$ Cluster of Pyruvate Formate-Lyase Activating Enzyme Generates the Glycyl Radical on Pyruvate Formate-Lyase: EPRDetected Single Turnover. J. Am. Chem. Soc. 122, 8331-8332.

(50) Massey, V., Hemmerich, P., Knappe, W. R., Duchstein, H. J., and Fenner, H. (1978) Photoreduction of flavoproteins and other biological compounds catalyzed by deazaflavins. Biochemistry 17, 9-16.

(51) Toy, A. D., Chaston, S. H. H., Pilbrow, J. R., and Smith, T. D. (1971) Electron Spin Resonance Study of Copper(II) Chelates of Certain Monothio-Beta-Diketones and Diethyldithiocarbamate. Inorg. Chem. 10, 2219-2225.

(52) Rakowsky, M. H., Zecevic, A., Eaton, G. R., and Eaton, S. S. (1998) Determination of high-spin iron(III)-nitroxyl distances in spinlabeled porphyrins by time-domain EPR. J. Magn. Reson. 131, 97-110.

(53) Eaton, S. S., and Eaton, G. R. (2002) Relaxation times of organic radicals and transition metal ions. Biol. Magn. Reson. 19, 29-154.

(54) Mulder, D. W., Ortillo, D. O., Gardenghi, D. J., Naumov, A. V., Ruebush, S. S., Szilagyi, R. K., Huynh, B., Broderick, J. B., and Peters, J. W. (2009) Activation of HydA(DeltaEFG) requires a preformed [4Fe4S] cluster. Biochemistry 48, 6240-6248.

(55) Mulder, D. W., Boyd, E. S., Sarma, R., Lange, R. K., Endrizzi, J. A., Broderick, J. B., and Peters, J. W. (2010) Stepwise [FeFe]hydrogenase $\mathrm{H}$-cluster assembly revealed in the structure of HydA(DeltaEFG). Nature 465, 248-251.

(56) Czech, I., Stripp, S., Sanganas, O., Leidel, N., Happe, T., and Haumann, M. (2011) The $[\mathrm{FeFe}]$-hydrogenase maturation protein HydF contains a H-cluster like [4Fe4S]-2Fe site. FEBS Lett. 585, 225230.

(57) Bertrand, P., Gayda, J. P., Fee, J. A., Kuila, D., and Cammack, R. (1987) Comparison of the spin-lattice relaxation properties of the two classes of $[2 \mathrm{Fe}-2 \mathrm{~S}]$ clusters in proteins. Biochim. Biophys. Acta, Protein Struct. Mol. Enzymol. 916, 24-28.

(58) Sarewicz, M., Dutka, M., Pietras, R., Borek, A., and Osyczka, A. (2015) Effect of $\mathrm{H}$ bond removal and changes in the position of the iron-sulphur head domain on the spin-lattice relaxation properties of the $[2 \mathrm{Fe}-2 \mathrm{~S}]^{2+}$ Rieske cluster in cytochrome bc(1). Phys. Chem. Chem. Phys. 17, 25297-25308.

(59) Usselman, R. J., Fielding, A. J., Frerman, F. E., Watmough, N. J., Eaton, G. R., and Eaton, S. S. (2008) Impact of mutations on the midpoint potential of the $[4 \mathrm{Fe}-4 \mathrm{~S}]^{+1,+2}$ cluster and on catalytic activity in electron transfer flavoprotein-ubiquinone oxidoreductase (ETFQO). Biochemistry 47, 92-100. 
(60) Broderick, J. B., Duderstadt, R. E., Fernandez, D. C., Wojtuszewski, K., Henshaw, T. F., and Johnson, M. K. (1997) Pyruvate formate-lyase activating enzyme is an iron-sulfur protein. $J$. Am. Chem. Soc. 119, 7396-7397.

(61) Krebs, C., Henshaw, T. F., Cheek, J., Huynh, B. H., and Broderick, J. B. (2000) Conversion of $3 \mathrm{Fe}-4 \mathrm{~S}$ to $4 \mathrm{Fe}-4 \mathrm{~S}$ clusters in native pyruvate formate-lyase activating enzyme: Mössbauer characterization and implications for mechanism. J. Am. Chem. Soc. 122, 1249712506.

(62) Walsby, C. J., Ortillo, D. O., Yang, J., Nnyepi, M. R., Broderick, W. E., Hoffman, B. M., and Broderick, J. B. (2005) Spectroscopic approaches to elucidating novel iron-sulfur chemistry in the "radicalSAM" protein superfamily. Inorg. Chem. 44, 727-741.

(63) Nicolet, Y., Rubach, J. K., Posewitz, M. C., Amara, P., Mathevon, C., Atta, M., Fontecave, M., and Fontecilla-Camps, J. C. (2008) X-ray structure of the $[\mathrm{FeFe}]$-hydrogenase maturase HydE from Thermotoga maritima. J. Biol. Chem. 283, 18861-18872.

(64) Nicolet, Y., Rohac, R., Martin, L., and Fontecilla-Camps, J. C. (2013) X-ray snapshots of possible intermediates in the time course of synthesis and degradation of protein-bound $\mathrm{Fe}_{4} \mathrm{~S}_{4}$ clusters. Proc. Natl. Acad. Sci. U. S. A. 110, 7188-7192.

(65) Gayda, J. P., Bertrand, P., Deville, A., More, C., Roger, G., Gibson, J. F., and Cammack, R. (1979) Temperature dependence of the electronic spin-lattice relaxation time in a 2-iron-2-sulfur protein. Biochim. Biophys. Acta, Protein Struct. 581, 15-26.

(66) Bertrand, P., Gayda, J. P., and Rao, K. K. (1982) Electron spinlattice relaxation of the $4 \mathrm{Fe}-4 \mathrm{~S}$ ferredoxin from Bacillus stearothermophilus. Comparison with other iron proteins. J. Chem. Phys. 76, 47154719 .

(67) Blum, H., Cusanovich, M. A., Sweeney, W. V., and Ohnishi, T. (1981) Magnetic interactions between dysprosium complexes and two soluble iron-sulfur proteins. J. Biol. Chem. 256, 2199-2206.

(68) Rupp, H., de la Torre, A., and Hall, D. O. (1979) The electron spin relaxation of the electron acceptors of photosystem I reaction centre studied by microwave power saturation. Biochim. Biophys. Acta, Bioenerg. 548, 552-564.

(69) Bertrand, P., Roger, G., and Gayda, J. P. (1980) Measurement of the spin-lattice relaxation time from the broadening of the EPR spectrum of a randomly-oriented system with $S=1 / 2$ : Application to iron-sulfur proteins. J. Magn. Reson. 40, 539-549.

(70) Gayda, J. P., Bertrand, P., Guigliarelli, B., and Meyer, J. (1983) About the structure of the three iron clusters in iron-sulfur proteins: An EPR comparison of Azotobacter vinelandii Fd I and Desulfovibrio gigas Fd II. J. Chem. Phys. 79, 5732-5733.

(71) Kuchenreuther, J. M., Britt, R. D., and Swartz, J. R. (2012) New insights into $[\mathrm{FeFe}]$ hydrogenase activation and maturase function. PLoS One 7, e45850.

(72) Sato, H., Kathirvelu, V., Fielding, A. J., Bottle, S. E., Blinco, J. P., Micallef, A. S., Eaton, S. S., and Eaton, G. R. (2007) Impact of molecular size on electron spin relaxation rates of nitroxyl radicals in glassy solvents between 100 and $300 \mathrm{~K}$. Mol. Phys. 105, 2137-2151.

(73) Zhou, Y., Bowler, B. E., Lynch, K., Eaton, S. S., and Eaton, G. R. (2000) Interspin distances in spin-labeled metmyoglobin variants determined by saturation recovery EPR. Biophys. J. 79, 1039-1052.

(74) Ulyanov, D., Bowler, B. E., Eaton, G. R., and Eaton, S. S. (2008) Electron-Electron Distances in Spin-Labeled Low-Spin Metmyoglobin Variants by Relaxation Enhancement. Biophys. J. 95, 5306-5316.

(75) Fielding, A. J., Usselman, R. J., Watmough, N., Simkovic, M., Frerman, F. E., Eaton, G. R., and Eaton, S. S. (2008) Electron spin relaxation enhancement measurements of interspin distances in human, porcine, and Rhodobacter electron transfer flavoproteinubiquinone oxidoreductase (ETF-QO). J. Magn. Reson. 190, 222-232.

(76) Shepard, E. M., Byer, A. S., Boyd, E. S., Swanson, K. D., Peters, J. W., and Broderick, J. B. (2013) [FeFe]-hydrogenase cofactor assembly. In Metals in Cells: Encyclopedia of Inorganic and Bioinorganic Chemistry (Culotta, V., and Scott, R. A., Eds.) John Wiley \& Sons, Chichester, U.K.

(77) Berto, P., Di Valentin, M., Cendron, L., Vallese, F., Albertini, M., Salvadori, E., Giacometti, G. M., Carbonera, D., and Costantini, P.
(2012) The [4Fe-4S]-cluster coordination of [FeFe]-hydrogenase maturation protein $\mathrm{HydF}$ as revealed by EPR and HYSCORE spectroscopies. Biochim. Biophys. Acta, Bioenerg. 1817, 2149-2157.

(78) Joshi, N., Shepard, E. M., Byer, A. S., Swanson, K. D., Broderick, J. B., and Peters, J. W. (2012) Iron-sulfur cluster coordination in the $[\mathrm{FeFe}]$-hydrogenase H-cluster biosynthetic factor HydF. FEBS Lett. 586, 3939-3943.

(79) Albertini, M., Berto, P., Vallese, F., Di Valentin, M., Costantini, P., and Carbonera, D. (2015) Probing the Solvent Accessibility of the [4Fe-4S] Cluster of the Hydrogenase Maturation Protein HydF from Thermotoga neapolitana by HYSCORE and 3p-ESEEM. J. Phys. Chem. B 119, 13680-13689.

(80) Berggren, G., Garcia-Serres, R., Brazzolotto, X., Clemancey, M., Gambarelli, S., Atta, M., Latour, J. M., Hernandez, H. L., Subramanian, S., Johnson, M. K., and Fontecave, M. (2014) An EPR/HYSCORE, Mössbauer, and resonance Raman study of the hydrogenase maturation enzyme HydF: a model for N-coordination to [4Fe-4S] clusters. J. Biol. Inorg. Chem. 19, 75-84. 